This is an Accepted Manuscript of an article published by Taylor \& Francis Group in Combustion Theory and Modelling on 01 Jun 2019, available online at: http://www.tandfonline.com/10.1080/13647830.2019.1600722

\title{
Effects of turbulencechemistry interactions on auto-ignition and flame structure for $n$-dodecane spray combustion
}

\author{
Yan Zhang ${ }^{\mathrm{a}}$, Hu Wang ${ }^{\mathrm{a}}$, Ambrus Both ${ }^{\mathrm{b}}$, Likun $\mathrm{Ma}^{\mathrm{c}}$ and Mingfa $\mathrm{Yao}^{\mathrm{a}}$

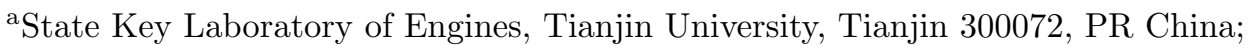 \\ ${ }^{\mathrm{b}}$ Computer Applications in Science and Engineering, Barcelona Supercomputing Center \\ (BSC), Barcelona 08006, Spain; ${ }^{\mathrm{C} C o l l e g e ~ o f ~ A e r o s p a c e ~ S c i e n c e ~ a n d ~ T e c h n o l o g y, ~ N a t i o n a l ~}$ \\ University of Defense Technology, Changsha 410073, PR China
}

\section{ARTICLE HISTORY}

Compiled June 8, 2020

\begin{abstract}
The Engine Combustion Network (ECN) Spray A under diesel engine conditions is investigated with a non-adiabatic 5D Flamelet Generated Manifold (FGM) model with the consideration of detailed chemical kinetic mechanisms. The enthalpy deficit due to droplet vaporization is considered by employing an additional controlling parameter in the FGM library. In this FGM model, $\beta$-PDF is used for the PDF integration over the control variable space. Validation results in non-reacting conditions indicate relatively good agreement between the predicted and experimental data in terms of liquid and vapor penetrations and mixture fraction spatial distribution. In reacting conditions, the effects of variance of mixture fraction and progress variable were examined. The ignition delay time and the quasi-steady flame structure are both affected by the variances. The variance of mixture fraction delays the ignition process and the variance of progress variable accelerates it. For mixture fraction, the ignition process is quicker at any stage in the case of neglecting variance. While things are more complex for progress variable, the ignition process is advanced in the case of neglecting variance at early times, but surpassed by the case of $\beta$-PDF later and until auto-ignition. When variance of mixture fraction is considered, the $\mathrm{OH}$ mass fraction shows a wide spatial distribution. While if not, a very thin flame is observed with a higher peak in $\mathrm{OH}$, and a very large lift-off length. The variance of progress variable has little impact on the global flame structure, but makes the flame lift-off length much shorter. This study confirms the general observation, that the variance of mixture fraction is of higher importance in high temperature nonpremixed combustion, however we found that the variance of progress variable is far from negligible.
\end{abstract}

\section{KEYWORDS}

Flamelet Generated manifolds; Turbulence-Chemistry Interaction; Enthalpy deficit; Engine Combustion Network ; Spray A

Yan Zhang. Email: zytju@tju.edu.cn

Hu Wang. Email: wang_hu@tju.edu.cn

Ambrus Both. Email: ambrus.both@bsc.es

Likun Ma. Email: malikun@nudt.edu.cn

Corresponding Author: Mingfa Yao. Email: y_mingfa@tju.edu.cn 


\section{Introduction}

Spray combustion is an important and challenging topic in internal combustion engine research. The focus of this work is diesel spray combustion which is characterized by high temperature non-premixed combustion[1]. In contrast to conventional diesel combustion, modern compression-ignition engines are characterized by low temperature combustion (LTC), with the purpose of reducing soot and $\mathrm{NO}_{\mathrm{x}}$ emissions. In this scenario, determining the global combustion phasing, auto-ignition is a strong function of the chemical kinetics and turbulence-chemistry interaction (TCI) [2]. To improve the understanding and design of internal combustion engines, it is essential to account for detailed chemistry mechanisms along with TCI effects, for the reasons discussed below. Accounting for detailed chemistry is crucial to accurately model the non-premixed spray auto-ignition and oxidation processes and also the pollutant emissions, in particular the evolution of PAH species [3]. On the other hand, as the Arrhenius chemical reaction rates are highly non-linear, the averaged reaction rate cannot be expressed as a function of the cell-averaged temperature, pressure, and species concentration [3]. Dahms et al. [4] reported that neglect of TCI fails to capture the key features of spray combustion ignition process. So in addition to detailed chemistry mechanisms, it is necessary to account for the effect of TCI to improve the accuracy of calculations in combustion models.

Many TCI models have been applied for the modeling of spray flames. Such as, the transported probability density function (TPDF) method $[5,6]$, the Representative Interactive Flamelet (RIF) [7, 8], the Flamelet/Progress Variable model (FPV) [9], the Flamelet-Generated Manifolds (FGM) $[10,11,12]$ and the Tabulated Flamelet Model (TFM) $[2,13,14]$. Dahms et al. [4] solved the 1D flamelet equations coupled with a detailed 2755 species n-dodecane mechanism [15] at the standard Spray A condition of the Engine Combustion Network (ECN) [16]. Compared to homogeneous reactor model, increase in scalar dissipation rate decreases the ignition delay and shifts the ignition location to richer regions. At higher scalar dissipation rate, however, the ignition delay retards. Pei et al. [5] carried out RANS simulations on spray A using the transported PDF method. It was found that the first stage ignition is initiated on the lean side and extremely resilient to turbulence. The second stage of ignition occurs first in rich mixtures and appears to be influenced strongly by turbulence. Relative to a homogeneous reactor, it is delayed on the lean side but advanced on the rich side, suggesting entrainment and mixing from the early igniting lean regions into richer mixtures is an important moderator of the ignition process. Another transported PDF simulation of spray A by Bolla et al. [6] shows that neglecting turbulent fluctuations results in an increase of the NO mass by a factor of two. Well-mixed model and multiple Representative Interactive Flamelets (mRIF) adopted on spray A are compared in [8]. Neglecting the turbulencechemistry interaction makes the flame very thin and causes a higher peak mean value of temperature and $\mathrm{OH}$ mass fraction. These findings are similar to those obtained with other non-premixed turbulent combustion models in which the turbulencechemistry interaction is taken into account $[10,17,7,18]$. Wehrfritz et al. [11] implemented the FGM combustion modeling approach to model the ECN spray A over a range of oxygen concentrations. Two different n-dodecane detailed chemistry mechanisms were compared with respect to auto-ignition, flame stabilization and species formation. Qualitative results showed good agreement between simulation and experiments with respect to formation of $\mathrm{CH}_{2} \mathrm{O}$.

Among these turbulent combustion models, flamelet based model is characterized by high computational efficiency, yet it can take detailed chemical kinetics into account. 
Wehrfritz et al. [11] used two n-dodecane mechanisms by Ranzi et al. [19] (130 species) and Narayanaswamy et al. [20] (257 species) with a LES-FGM framework. Kundu et al. [14] developed a new framework with the LSODES (Livermore Solver for ODEs in Sparse form) [21] chemistry solver and an analytical Jacobian to calculate 1D flamelets with detailed 2755 species n-dodecane mechanism [15], and adopted them into TFM simulation with LES. According to Kundu's study, the detailed mechanism performs better at the lower temperature conditions where low temperature reactions play important roles, compared to a reduced mechanism with 103 species [22]. The basic idea of flamelet approaches [23] is that a multi-dimensional turbulent flame may be considered as an ensemble of stretched one-dimensional laminar flames, called flamelets, embedded within the turbulent flow field. Mixture fraction $Z$ is introduced to eliminate the need for non-linear chemical source term. Consequently, the chemistry can be solved in the mixture fraction coordinate and then mapped back to the flow field. Flamelet based models combined with tabulated chemistry methods reduce the computational cost by decoupling 3D-CFD and laminar flamelet calculations. This has enabled the flamelet models to use large chemistry mechanisms with relatively lower computational cost. Additionally, flamelet based models are able to account for the turbulence-chemistry interaction (TCI) efficiently by means of presumed probability density functions. Flamelet assumption is valid only when the characteristic chemical time scales remain short in comparison to the mixing time scales $[24,3]$, as is the case for diesel-like combustion events in most of the relevant conditions [25]. Dahms [4] investigated auto-ignition process of spray A in 1D flamelets, which reveals that the high Damkhler number limit, a key requirement for the validity of the flamelet assumption, applies during the entire non-equilibrium ignition process of the Spray A conditions. Actually, the validity of flamelet model adopted on 3D non-premixed spray combustion modelling has already been verified $[2,7,8,9,10,11,12,13,14,26]$.

Although many researches have been done about TCI in computational modeling of the ECN Spray A, there are several unexplored questions, which are the main topics of the present paper. In the present paper, RANS modelling of ECN spray A with the fuel of $n$-Dodecane is carried out using FGM [27] combustion model to account for the turbulence-chemistry interaction along with complex chemical reactions. The effect of variance of mixture fraction has already been investigated $[10,8,7,18]$, with the conclusion of maximum temperature will be suppressed and $\mathrm{OH}$ becomes broader with the introduction of $Z^{\prime \prime 2}$, while lacking detailed explanation, which is one of topics in this study. In addition, variance of progress variable is neglected because of conventional wisdom that high temperature non-premixed combustion is mixing limited and it significantly relies on the rate of mixture formation, as reported in the previous literature $[10,11,12]$. However, it is well-known that a reactive scalar, such as $Y_{c}$, depends on a combination of solutions of laminar flamelet equation for each chemical state and therefore its PDF cannot be accurately approximated by a Dirac delta distribution [28]. Furthermore, since the trend of LTC, importance of chemistry should be taken into account in the combustion model. For these reasons, variance of progress variable was considered in the present work, similar treatment with progress variable in premixed systems [3].

The objectives of this study are to improve the understanding of the auto-ignition process, and to delineate the effect of variance of mixture fraction and progress on the auto-ignition process and the flame structure. The paper is organized as follows. The first part of this study describes the model formulation (Section 2). Then the experimental and simulation details are briefly described in Section 3. The results of the non-reacting cases are analyzed in Section 4.1. The pre-processing of FGM tables 
procedure is shown in Section 4.2. Then Section 4.3 gives the global view of ignition process. This is followed by the analysis the effects of variances of mixture fraction and progress variable on the ignition process and flame structure. A summary and conclusions are given in Section 5.

\section{Numerical methods}

\subsection{Gas phase models}

In the current study, the FGM model is adopted for the Turbulence-Chemistry Interaction (TCI), which was at first developed for premixed flames by van Oijen and Goey [27] and applied to non-premixed flames later [29]. The first application of the FGM model to the simulation of an igniting diesel spray was conducted by Bekdemir et al. [30]. The FGM model shares the idea with flamelet approaches that a multidimensional flame may be considered as an ensemble of one-dimensional flames. Although the interaction between diffusive layers such as in MILD (moderate or intense low-oxygen dilution) conditions will shorten the ignition delay [31], the igniting spray in the present paper can be seen as isolated flamelet structures. The FGM model is also characterized by the storage and retrieval procedure. Except for mixture fraction, another control variable the reaction progress variable is introduced to consider the unsteady process, which makes it possible to cover auto-ignition phenomenon in spray combustion. The FGM model used in this study can be summarized in the following steps:

(1) Calculation of the representative 1D flamelets.

(2) Transformation of 1D flamelets solutions to the control variable space.

(3) PDF integration by means of presumed PDF method.

(4) Storage of FGM tables as function of mean and variance values of the control variables.

(5) Retrieval of thermo-chemical variables from the FGM tables according to FGM control variables and their variances solved by $3 \mathrm{D}$ governing equations.

The Favre-filtered transport equations for turbulent spray combustion using FGM 
method can be expressed as follows:

$$
\begin{aligned}
& \frac{\partial \bar{\rho}}{\partial t}+\frac{\partial \bar{\rho} \widetilde{u_{i}}}{\partial x_{i}}=\overline{S_{\rho}} \\
& \frac{\partial}{\partial t}\left(\bar{\rho} \widetilde{u_{i}}\right)+\frac{\partial \bar{\rho} \widetilde{u_{i}} \widetilde{u_{j}}}{\partial x_{j}}=\frac{\partial \bar{\sigma}_{i j}}{\partial x_{j}}+\frac{\partial}{\partial x_{j}}\left(-\bar{\rho} \widetilde{u_{i}^{\prime} u_{j}^{\prime}}\right)+\overline{S_{u_{i}}} \\
& \frac{\partial}{\partial t}(\bar{\rho} \widetilde{h})+\frac{\partial \bar{\rho} \widetilde{u}_{i} \widetilde{h}}{\partial x_{i}}=\frac{D \bar{p}}{D t}+\frac{\partial}{\partial x_{i}}\left(\alpha_{e f f} \frac{\partial \widetilde{h}}{\partial x_{i}}\right)+\overline{S_{h}} \\
& \frac{\partial}{\partial t}(\bar{\rho} \widetilde{Z})+\frac{\partial \bar{\rho} \widetilde{u_{i}} \widetilde{Z}}{\partial x_{i}}=\frac{\partial}{\partial x_{i}}\left[\left(\frac{\mu}{S c}+\frac{\mu_{t}}{S c_{t}}\right) \frac{\partial \widetilde{Z}}{\partial x_{i}}\right]+\overline{S_{Z}} \\
& \frac{\partial}{\partial t}\left(\bar{\rho} \widetilde{Y}_{c}\right)+\frac{\partial \bar{\rho} \widetilde{u}_{i} \widetilde{Y}_{c}}{\partial x_{i}}=\frac{\partial}{\partial x_{i}}\left[\left(\frac{\mu}{S c}+\frac{\mu_{t}}{S c_{t}}\right) \frac{\partial \widetilde{Y}_{c}}{\partial x_{i}}\right]+\overline{\dot{\omega}_{Y_{c}}} \\
& \frac{\partial}{\partial t}\left(\bar{\rho} \widetilde{Z^{\prime \prime 2}}\right)+\frac{\partial \bar{\rho} \widetilde{u_{i}} \widetilde{Z^{\prime \prime 2}}}{\partial x_{i}}=\frac{\partial}{\partial x_{i}}\left[\left(\frac{\mu}{S c}+\frac{\mu_{t}}{S c_{t}}\right) \frac{\partial \widetilde{Z^{\prime \prime 2}}}{\partial x_{i}}\right] \\
& +C_{g} \frac{\mu_{t}}{S c_{t}}\left(\frac{\partial \widetilde{Z}}{\partial x_{i}}\right)^{2}-C_{d, Z v} \bar{\rho} \widetilde{S_{\chi_{z}}}+\alpha_{Z v} \widetilde{Z^{\prime \prime 2}}\left(\frac{\overline{S_{Z}}}{\widetilde{Z}}\right) \\
& \frac{\partial}{\partial t}\left(\widetilde{\rho} \widetilde{Y_{c}^{\prime \prime 2}}\right)+\frac{\partial \bar{\rho} \widetilde{u_{i}} \widetilde{Y_{c}^{\prime \prime 2}}}{\partial x_{i}}=\frac{\partial}{\partial x_{i}}\left[\left(\frac{\mu}{S c}+\frac{\mu_{t}}{S c_{t}}\right) \frac{\partial \widetilde{Y_{c}^{\prime \prime 2}}}{\partial x_{i}}\right] \\
& +C_{g} \frac{\mu_{t}}{S c_{t}}\left(\frac{\partial \widetilde{Y}_{c}}{\partial x_{i}}\right)^{2}-C_{d, Y_{c} v} \bar{\rho} \widetilde{S_{\chi_{Y_{c}}}}+2\left(\overline{Y_{c} \dot{\omega}_{Y_{c}}}-\widetilde{Y}_{c} \overline{\dot{\omega}_{Y_{c}}}\right),
\end{aligned}
$$

where $\bar{\rho}, \widetilde{u_{i}}, \bar{p}, \widetilde{h}, \widetilde{Z}, \widetilde{Y_{c}}, \widetilde{Z^{\prime \prime 2}}, \widetilde{Y_{c}^{\prime \prime 2}}$, denote the filtered density, velocity, pressure, absolute enthalpy, mixture fraction, reaction progress variable, variance of mixture fraction, and variance of reaction progress variable respectively. $\overline{S_{\rho}}, \overline{S_{u_{i}}}, \overline{S_{h}}, \overline{S_{Z}}$ are respectively the source term for density, momentum, absolute enthalpy and mixture fraction due to droplet evaporation. The tilde $\widetilde{\phi}$ denotes Favre average of variable $\phi$ while bar $\bar{\phi}$ denotes Reynolds average. These 9 transport equations are closed by the equation of state of ideal gas: $p=\rho R_{u} / W T\left(R_{u}=8.314 \mathrm{~J} /(\mathrm{mol} \cdot \mathrm{K}), W\right.$ is molecular weight of mixture). The other variables in the transport equations can be expressed as function of the unknowns. Viscous and pressure tensors are often combined into the $\sigma_{i j}$ tensor defined by:

$$
\sigma_{i j}=\tau_{i j}-p \delta_{i j}=2 \mu\left(S_{i j}-\frac{1}{3} S_{k k} \delta_{i j}\right)-p \delta_{i j}
$$

where the strain rate is $S_{i j}=\frac{1}{2}\left(\frac{\partial u_{i}}{\partial x_{j}}+\frac{\partial u_{j}}{\partial x_{i}}\right) \cdot R_{i j}=-\bar{\rho} \widetilde{u_{i}^{\prime} u_{j}^{\prime}}$ is Reynolds stress, according to Boussinesqs hypothesis [32]: $R_{i j}-\frac{1}{3} R_{k k} \delta_{i j}=2 \mu_{t}\left(\widetilde{S_{i j}}-\frac{1}{3} \widetilde{S_{k k}} \delta_{i j}\right) . \mu_{t}$ in the $k-\epsilon$ model is calculated as $\mu_{t}=\rho C_{\mu} k^{2} / \epsilon$, where $C_{\mu}$ is model constant. The effective thermal diffusivity of mixture is calculated as: $\alpha_{e f f}=\alpha+\alpha_{t}, \alpha$ is interpolated from FGM tables and $\alpha_{t}=\mu_{t} / \operatorname{Pr}_{t} . S c$ and $S c_{t}$ are laminar and turbulent Schmidt number respectively. $C_{g}, C_{d, Z v}, C_{d, Y_{c} v}$ are modeling constants for the generation and dissipation terms of mixture fraction and progress variable variances respectively, with the theoretical value of 2 . Model constant value $\alpha_{Z v}=0.5$ is used in the current study. The turbulent scalar dissipation rate of mixture fraction and progress variable are modeled assuming 
the time scale of dissipation of the scalar variances is the same as the time scale of turbulence $\left(\tau_{t}=k / \epsilon\right)$ :

$$
\begin{aligned}
& \bar{\rho} \widetilde{S_{\chi Z}}=\bar{\rho} \widetilde{\chi_{Z}}-\bar{\rho} \widetilde{D}\left(\frac{\partial \widetilde{Z}}{\partial x_{j}}\right)^{2}=\bar{\rho} \frac{\epsilon}{k} \widetilde{Z^{\prime \prime 2}} \\
& \bar{\rho} \widetilde{S_{\chi Y_{c}}}=\bar{\rho} \widetilde{\chi_{Y_{c}}}-\bar{\rho} \widetilde{D}\left(\frac{\partial \widetilde{Y}_{c}}{\partial x_{j}}\right)^{2}=\bar{\rho} \frac{\epsilon}{k} \widetilde{Y_{c}^{\prime \prime 2}}
\end{aligned}
$$

Table 1. Source terms from Lagrangian

\begin{tabular}{ll}
\hline source term & Expression \\
\hline$\overline{S_{\rho}}$ & $-\frac{1}{V_{c}} \sum_{p} \dot{m}_{p} N_{p}$ \\
$\overline{S_{u_{i}}}$ & $-\frac{1}{V_{c}} \sum_{p} \dot{m}_{p} N_{p}\left[\left(U_{p, i}^{t_{n}+\Delta t}-U_{p, i}^{t_{n}}\right) / \Delta t-g_{i}\right]-\frac{1}{V_{c}} \sum_{p} \dot{m}_{p} N_{p} U_{p, i}^{t_{n}}$ \\
$\overline{S_{h}}$ & $\frac{1}{V_{c}} \sum_{p} N_{p}\left(T_{p}-T_{c}\right) \pi D_{p} \lambda_{m} N u_{m}-\frac{1}{V_{c}} \sum_{p} \dot{m}_{p} N_{p} h\left(T_{p}\right)$ \\
$\overline{S_{Z}}$ & $-\frac{1}{V_{c}} \sum_{p} \dot{m}_{p} N_{p}$ \\
\hline
\end{tabular}

The source terms of gas phase governing equations from Lagrangian droplets are presented in Table 1, where the number of droplets represented by a parcel is $N_{p}$, the volume of the computational cell containing the parcel is $V_{c}$, the $t_{n}$ and $t_{n}+\Delta t$ superscripts mark the old and new time steps, and $h\left(T_{p}\right)$ is the absolute enthalpy of the evaporated fuel.

\subsection{Turbulence chemistry interaction}

In this work Bilger's formula [33] is used to calculate the mixture fraction.

$$
Z=\frac{2 \frac{Y_{C}-Y_{C, 2}}{W_{C}}+0.5 \frac{Y_{H}-Y_{H, 2}}{W_{H}}-\frac{Y_{O}-Y_{O, 2}}{W_{O}}}{2 \frac{Y_{C, 1}-Y_{C, 2}}{W_{C}}+0.5 \frac{Y_{H, 1}-Y_{H, 2}}{W_{H}}-\frac{Y_{O, 1}-Y_{O, 2}}{W_{O}}}
$$

Above, $Y$ and $W$ are the mass fraction and molecular mass, respectively. Subscripts $C, H, O$ refer to the carbon, hydrogen and oxygen elements and subscripts 1,2 refer the pure fuel and pure oxidizer, respectively.

The reaction progress variable $Y_{c}$ is commonly defined from a combination of reactive scalars such as chemical species or temperature. Although its definition is not unique, suggested by Ihme et al. [34], the prerequisite for the definition of a suitable reaction progress variable is that the set of parameters from which the manifold is formed should uniquely characterize each point in the thermo-chemical state-space. That is to say, the reaction progress variable should be injective from unburnt conditions to equilibrium. The present reaction progress variable definition is chosen to represent the first onset of ignition via $\mathrm{HO}_{2}$ contribution and to progress towards the steady flame via $\mathrm{CO}$ and $\mathrm{CO}_{2}$, the same definition is chosen by Bekdemir et al. $[35,36]$ and Egüz et al. [10], for the modelling of n-heptane spray auto-ignition and steady diffusion flame.

$$
Y_{c}=\frac{Y_{C O_{2}}}{M_{C O_{2}}}+\frac{Y_{C O}}{M_{C O}}+\frac{Y_{\mathrm{HO}_{2}}}{M_{\mathrm{HO}_{2}}}
$$

The choice for a combination of $\mathrm{CO}, \mathrm{CO}_{2}$ and $\mathrm{HO}_{2}$ mass fractions stems from the fact that the ignition in low temperature oxidation starts with $\mathrm{HO}_{2}$, being the first 
species formed from fuel and oxygen, experiment evidence by Blocquet et al. [37]. Soon thereafter, $\mathrm{CO}$ starts to take over and finally converts to $\mathrm{CO}_{2}$. The monotonicity of the progress variable is ensured when constructing the manifold, leading to a consistent FGM tables. Since in non-premixed combustion $Y_{c}$ is dependent on mixture fraction $Z$, a normalized progress variable $C$ is defined as:

$$
C\left(Y_{c}, Z\right)=\frac{Y_{c}-Y_{c}^{u}(Z)}{Y_{c}^{b}(Z)-Y_{c}^{u}(Z)}
$$

where $Y_{c}^{u}(Z)$ and $Y_{c}^{b}(Z)$ denote the minimum and maximum values of $Y_{c}$ at a given mixture fraction.

The influence of turbulence fluctuations on the local flame structure is accounted through the joint PDF of the independent variables. The Favre-averaged/filtered scalars $\phi$ (e.g. $T, Y_{i}$, etc), are expressed as:

$$
\widetilde{\phi}=\int_{0}^{1} \int_{0}^{1} \phi(Z, C) \widetilde{P}(Z, C) d Z d C
$$

The Reynolds-averaged/filtered mean of scalar $\bar{\phi}$ and density $\bar{\rho}$ can be computed as follows:

$$
\begin{aligned}
& \bar{\phi}=\bar{\rho} \int_{0}^{1} \frac{\phi(Z, C)}{\rho(Z, C)} \widetilde{P}(Z, C) d Z d C \\
& \bar{\rho}=\left[\int_{0}^{1} \frac{\widetilde{P}(Z, C)}{\rho(Z, C)}\right]^{-1}
\end{aligned}
$$

It is a common practice to assume that $Z$ and $C$ are statistically independent of each other, then the joint PDF can be computed as the product of marginal PDFs:

$$
\widetilde{P}(Z, C)=\widetilde{P}(Z) \widetilde{P}(C)
$$

A posteriori analysis of DNS of spray combustion [38] found that $\beta$-function PDF is a good representation for the mixture fraction distribution. They investigated three cases at three different timings, and found all the predictions by presumed $\beta$-PDF are in excellent agreement with the statistics results of DNS. And $\beta$-PDF is also a widely employed choice in the related studies $[2,7,8,9,10,11,12,13,14,18]$. Mukhopadhyay et al. [39] assessed the accuracy of such model in predicting autoignition and flame development in compositionally stratified n-heptane/air mixtures using direct numerical simulations (DNS). They found that $\beta$-PDF are able to reasonably approximate the actual PDF. The $\beta$-function PDF of scalar $\phi$ is expressed as:

$$
\widetilde{P}(\phi)=\frac{\phi^{\alpha-1}(1-\phi)^{\beta-1} \Gamma(\alpha+\beta)}{\Gamma(\alpha) \Gamma(\beta)},
$$

where the shape parameters $\alpha$ and $\beta$ are determined by mean and variance of scalar $\phi$ :

$$
\alpha=\widetilde{\phi}\left[\frac{\widetilde{\phi}(1-\widetilde{\phi})}{\widetilde{\phi^{\prime \prime 2}}}\right], \beta=(1-\widetilde{\phi}) \frac{\alpha}{\widetilde{\phi}}
$$


In this study, control variables are $Z$ and $C$, and are both integrated by $\beta$-function $\mathrm{PDF}$, which means that their mean values $\widetilde{Z}, \widetilde{C}$ and variances $\widetilde{Z^{\prime \prime 2}}, \widetilde{C^{\prime \prime 2}}$ are all included. $\widetilde{Z}$ and $\widetilde{Z^{\prime \prime 2}}$ are solved in transport equations, which also solve $\widetilde{Y}_{c}$ and $\widetilde{Y_{c}^{\prime \prime 2}}$. Normalization of $\widetilde{Y}_{c}$ leads to $\widetilde{C}$ :

$$
\widetilde{C}\left(\widetilde{Y_{c}}, \widetilde{Z}, \widetilde{Z^{\prime \prime 2}}\right)=\frac{\widetilde{Y_{c}}-\widetilde{Y_{c}^{u}}\left(\widetilde{Z}, \widetilde{Z^{\prime \prime 2}}\right)}{\widetilde{Y_{c}^{b}}\left(\widetilde{Z}, \widetilde{Z^{\prime \prime 2}}\right)-\widetilde{Y_{c}^{u}}\left(\widetilde{Z}, \widetilde{Z^{\prime \prime 2}}\right)},
$$

where $\widetilde{Y_{c}^{u}}$ and $\widetilde{Y_{c}^{b}}$ are the minimum and maximum progress variable values respectively, and stored in the 2D-FGM tables which the dimensions are mixture fraction and its variance. Conversion of $Y_{c}^{\prime \prime 2}$ to $C^{\prime \prime 2}$ can be done using:

$$
\widetilde{C^{\prime \prime 2}}=\frac{\widetilde{Y_{c}^{\prime \prime 2}}+\widetilde{Y}_{c}^{2}-\widetilde{\left(Y_{c}^{u}\right)^{2}}-2 \widetilde{C}\left(\widetilde{Y_{c}^{b} Y_{c}^{u}}-\widetilde{\left(Y_{c}^{u}\right)^{2}}\right)}{\widetilde{\left(Y_{c}^{b}\right)^{2}}-2 \widetilde{Y_{c}^{b} Y_{c}^{u}}+\widetilde{\left(Y_{c}^{u}\right)^{2}}}-\widetilde{C}^{2}
$$

where $\widetilde{\left(Y_{c}^{u}\right)^{2}}=\widetilde{\left(Y_{c}^{u}\right)^{2}}\left(\widetilde{Z}, \widetilde{Z^{\prime \prime 2}}\right), \widetilde{\left(Y_{c}^{b}\right)^{2}}=\widetilde{\left(Y_{c}^{b}\right)^{2}}\left(\widetilde{Z}, \widetilde{Z^{\prime \prime 2}}\right)$ and $\widetilde{Y_{c}^{b} Y_{c}^{u}}=\widetilde{Y_{c}^{b} Y_{c}^{u}}\left(\widetilde{Z}, \widetilde{Z^{\prime \prime 2}}\right)$ are stored in the 2D-FGM tables with the same dimensions of $\widetilde{Y_{c}^{u}}$ and $\widetilde{Y_{c}^{b}}$.

The range of variance depends on the mean value, therefore to simplify the table construction, the scaled variances of mixture fraction and progress variable $\widetilde{\zeta_{Z}}$ and $\widetilde{\zeta_{C}}$ are used as controlling parameters in the lookup table.

$$
\begin{aligned}
\widetilde{\zeta_{Z}} & =\frac{\widetilde{Z^{\prime \prime 2}}}{\widetilde{Z}(1-\widetilde{Z})} \\
\widetilde{\zeta_{C}} & =\frac{\widetilde{C^{\prime \prime 2}}}{\widetilde{C}(1-\widetilde{C})}
\end{aligned}
$$

\subsection{Enthalpy deficit in FGM}

To use FGM model in spray combustion modeling, the cooling effect due to spray evaporation and consequent reduction effect of the lower temperature on the chemistry reaction rate must be considered. In order to take into account the enthalpy deficit effect, an additional control variable was added to the FGM libraries in the present paper. The implementation of the enthalpy deficit effect procedure consists of two steps: 1. creating the FGM libraries with different levels of heat loss (different air side temperature in the current study), and parameterizing these libraries with the additional parameter. 2. obtaining this parameter during the turbulent combustion simulation, and using it for table lookup. In the current study, the normalized enthalpy deficit $\eta_{h}[40]$ is introduced to indicate the thermo-chemical space from the adiabatic state $\left(\eta_{h}=0\right)$ to the one with maximum heat loss $\left(\eta_{h}=1\right)$. The filtered normalized enthalpy deficit $\widetilde{\eta_{h}}$ is calculated as: $\widetilde{\eta_{h}}=\widetilde{d h} / \widetilde{d h_{\max }}$, where $\widetilde{d h}$ and $\widetilde{d h_{\max }}$ are the enthalpy difference between the adiabatic state and current state and the one with 
maximum enthalpy loss, under a given mixture fraction $\widetilde{Z}$.

$$
\begin{aligned}
& \widetilde{d h}=\widetilde{h}-\widetilde{h_{a d}}(\widetilde{Z}) \\
& \widetilde{d h_{\text {max }}}(\widetilde{Z})=(1-\widetilde{Z})\left(\left.h_{\text {min }}\right|_{Z=0}-\left.h_{a d}\right|_{Z=0}\right) \\
& \widetilde{h_{a d}}(\widetilde{Z})=\left.h_{a d}\right|_{Z=0}+\widetilde{Z}\left(\left.h_{a d}\right|_{Z=1}-\left.h_{a d}\right|_{Z=0}\right)
\end{aligned}
$$

With the additional dimension $\eta_{h}$, the FGM tables now depend on $Z, C$ and $\eta_{h}$ :

$$
\widetilde{\phi}=\int_{0}^{1} \int_{0}^{1} \int_{0}^{1} \phi\left(Z, C, \eta_{h}\right) \widetilde{P}\left(Z, C, \eta_{h}\right) d Z d C d \eta_{h}
$$

Statistical independence of the three controlling parameters is assumed:

$$
\widetilde{P}\left(Z, C, \eta_{h}\right)=\widetilde{P}(Z) \widetilde{P}(C) \widetilde{P}\left(\eta_{h}\right)
$$

$\beta$-function is employed for PDF of $Z$ and $C$, and $\delta$-function is used for PDF of $\eta_{h}$, such that the tabulated properties are:

$$
\widetilde{\phi}=\widetilde{\phi}\left(\widetilde{Z}, \widetilde{\zeta_{Z}}, \widetilde{C}, \widetilde{\zeta_{C}}, \widetilde{\eta_{h}}\right)
$$

Once the new FGM tables are generated, the CFD solver can evaluate normalized enthalpy deficit $\widetilde{\eta_{h}}$ to indicate the fifth dimension of FGM tables. However, $\widetilde{\eta_{h}}$ may be sometimes out of range [0-1], which requires additional consideration. $\widetilde{\eta_{h}}>1$ indicates that current enthalpy is below the lower bound of the state with maximum heat loss, which implys extra cooling effect. On the other hand, although it's usual for the case $\widetilde{d h}>0\left(\widetilde{h}>\widetilde{h_{a d}}\right), \widetilde{\eta_{h}}<0$ should be considered. On this occasion, enthalpy is been transferred from droplet to the gas environment. Given all of this, the thermochemical variables are interpolated from FGM tables by these five control parameters $\left(\widetilde{Z}, \widetilde{\zeta_{Z}}, \widetilde{C}, \widetilde{\zeta_{C}}, \widetilde{\eta_{h}}\right)$, and extra enthalpy due to $\widetilde{\eta_{h}}$ beyond $0-1$ is calculated as:

$$
d h_{\text {Extra }}= \begin{cases}\widetilde{d h}-\widetilde{d h_{\max }} & \widetilde{\eta_{h}}>1 \\ \widetilde{d h} & \widetilde{\eta_{h}}<0 \\ 0 & \text { else }\end{cases}
$$

which is used to correct the temperature with $d T=d h_{E x t r a} / C p$.

\subsection{Memory reduction}

The traditional FGM model retrieves species mass fraction from FGM tables, and then calculates mass-fraction-weighted average dynamic viscosity $\mu$ and diffusivity of enthalpy $\alpha$. Therefore, it is required to include all species in the FGM tables, which limits the adoption of detailed chemical mechanism, since it will consume an unsustainable amount of Random Access Memory (RAM). Wehrfritz et al. [11] and Kahila et al. [12] calculate $\mu$ with only several representative species to decrease the number of FGM tables. In the present study, $\mu$ and $\alpha$ are stored in the FGM tables and then retrieved by the CFD solver, so there is no need to include any species mass fraction in the FGM tables except the species used for post-processing. This method 
not only reduces the memory consumption in the CFD solver, but it also mitigates the computational load of the FGM table generation, which is often used by researchers whose interest lies in gas phase fuel combustion [41, 42].

However, in spray combustion modeling, species mass fractions are still required by the widely used heat and mass transfer models of the Lagrangian droplets. The properties at the mean conditions in the boundary layer $\rho_{m}, \mu_{m}, P r_{m}$ and $\kappa_{m}$ are used to evaluated mass and heat transfer between droplet phase and gas phase in the OpenFOAM Lagrangian library. At first, mean values $T_{m}$ and $Y_{k, m}$ between the particle surface state and the cell state are calculated by " $1 / 3$ rule".

$$
\begin{aligned}
& T_{m}=\left(2 T_{p}+T_{c}\right) / 3 \\
& Y_{k, m}=\left(2 Y_{k, s}+Y_{k, c}\right) / 3,
\end{aligned}
$$

where $T_{p}$ and $T_{c}$ are temperature of particle and cell respectively, and $Y_{k, s}$ and $Y_{k, c}$ are mass fraction of species $k$ on the droplet surface and in the cell. Then $\rho_{m}, \mu_{m}, \operatorname{Pr}_{m}$ and $\kappa_{m}$ are evaluated using the material property functions of the gas mixture at the state given by $\left(T_{m}, Y_{k, m}, p\right)$. The method introduced by Both [43] and used in this work omits the storage of species mass fractions in order to use arbitrarily large chemical mechanisms. The transport properties representative to the droplet boundary layer are directly retrieved from the FGM tables, instead of being calculated as a function of the mass fractions. The procedure is explained below. $Y_{k, n f, s}$ and $Y_{k, n f, c}$ are mass fraction of species $k$ excluding the fuel on the droplet surface and in the cell respectively.

$$
\begin{aligned}
Y_{k, n f, s} & =\frac{Y_{k, s}}{1-Y_{f, s}} \\
Y_{k, n f, c} & =\frac{Y_{k, c}}{1-Y_{f, c}},
\end{aligned}
$$

where $Y_{k, s}$ and $Y_{k, c}$ denote mass fraction of species k (any species other than fuel), $Y_{f, s}$ and $Y_{f, c}$ are mass fraction of fuel, subscripts s and $\mathrm{c}$ refer to the value on the droplet surface and in the cell respectively. The evaporation is driven by the concentration gradient of fuel in the boundary layer, thus in the presence of mass transfer the fuel mass fraction is different on the droplet surface and in the surrounding gas. In this work, we assume frozen chemistry in the droplet boundary layer, thus the constitution of the other species are identical on the droplet surface and in the surrounding cell, namely $Y_{k, n f, s}=Y_{k, n f, c}$, which yields the surface mass fraction of species other than fuel: $Y_{k, s}=Y_{k, n f, c}\left(1-Y_{f, s}\right)$. Since $Y_{c}$ is the combination of several mass fraction of species, the progress variable on the droplet surface can also be calculated in this way: $Y_{c, s}=Y_{c, n f, c}\left(1-Y_{f, s}\right)$, where $Y_{c, n f, c}$ is the progress variable of mixture in the cell excluding fuel, latter property is tabulated during the FGM generation.

The surface mixture fraction consists of the fuel mass fraction on the droplet surface, and the mixture fraction contribution of the non-fuel components, which is calculated as: $Z_{s}=Y_{f, s}+\left(1-Y_{f, s}\right) Z_{n f, c}$, where $Z_{n f, c}$ is the mixture fraction of the surrounding gas mixture excluding the fuel, according to Bilger's definition, calculated as: $Z_{n f, c}=$ $\left(Z_{c}-64.7139 Y_{f, c}\right) /\left(1-Y_{f, c}\right)$. The mixture fraction and progress variable variances on 
the droplet surface are calculated similarly with $Y_{c, s}$ :

$$
\begin{aligned}
& \widetilde{Z_{s}^{\prime \prime 2}}=\left(1-Y_{f, s}\right) \widetilde{Z_{c}^{\prime \prime 2}} \\
& \widetilde{Y_{c, s}^{\prime \prime 2}}=\left(1-Y_{f, s}\right) \widetilde{Y_{c, c}^{\prime \prime 2}}
\end{aligned}
$$

The enthalpy on the particle surface $\widetilde{h_{s}}$ are calculated using Newton-Raphson method keeping the other control variables constant, such that the surface temperature is equal to the droplet temperature $\left(T_{p}\right)$ :

$$
T\left(\widetilde{Z_{s}}, \widetilde{Y_{c, s}}, \widetilde{Z_{s}^{\prime \prime 2}}, \widetilde{Y_{c, s}^{\prime \prime}}, \widetilde{h_{s}}\right)=T_{p}
$$

The " $1 / 3$ rule" is applied to the control variables instead of the species:

$$
\begin{aligned}
& Z_{m}=\left(2 Z_{s}+Z_{c}\right) / 3 \\
& C_{m}=\left(2 C_{s}+C_{c}\right) / 3 \\
& Z_{m}^{\prime \prime 2}=\left(2 Z_{s}^{\prime \prime 2}+Z_{c}^{\prime \prime 2}\right) / 3 \\
& C_{m}^{\prime \prime 2}=\left(2 C_{s}^{\prime \prime 2}+C_{c}^{\prime \prime 2}\right) / 3 \\
& h_{m}=\left(2 h_{s}+h_{c}\right) / 3
\end{aligned}
$$

Using $\widetilde{Z_{m}}, \widetilde{C_{m}}, \widetilde{Z_{m}^{\prime \prime 2}}, \widetilde{C_{m}^{\prime \prime 2}}$, and $\widetilde{h_{m}}$ we retrieve material properties from the FGM tables: $\mu_{m}, \alpha_{m}, \psi_{m}$, and $C_{p, m}$, and calculate other properties: $\rho_{m}=p_{c} \psi_{m}, P r_{m}=\frac{\mu_{m}}{\alpha_{m}}$, $\kappa_{m}=\alpha_{m} C_{p, m}$.

Mass fraction of species are required in another place. The rate of evaporation for a single particle $\dot{m}_{p}$ is given by the expression:

$$
\dot{m}_{p}=\frac{d m_{p}}{d t}=-\pi D_{p} \mathcal{D} S h \rho_{m} \ln \left(1+B_{M}\right)
$$

where $D_{p}$ is particle diameter, $\mathcal{D}$ is mass diffusion coefficient of selected fuel, $S h$ is Sherwood number, calculated according to the Ranz and Marshall correlation [44, 45]: $S h=2+0.552 R e_{p}^{1 / 2} S c_{m}^{1 / 3}, \rho_{m}$ is the density of the fuel vapor in the droplet boundary layer. $B_{M}$ is Spalding number: $B_{M}=\frac{Y_{f, s}-Y_{f, c}}{1-Y_{f, s}}$, which requires mass fraction of fuel on the particle surface and in the cell too. The fuel mass fraction in the cell is tabulated, while the fuel mass fraction on the droplet surface is calculated using the mole fractions: $Y_{f, s}=\frac{X_{f, s} W_{f}}{X_{f, s} W_{f}+\left(1-X_{f, s}\right) / W_{n f, c}}$, where $X_{f, s}$ is mole fraction of fuel on the surface $X_{f, s}=p_{s a t} / p_{c}, W_{f}$ is molar mass of fuel, $W_{n f, c}$ is the average molar mass of the mixture excluding the fuel in the cell $W_{n f, c}=\left(1-Y_{f, c}\right) / \sum_{k=1, k \neq k_{f}}^{N_{s}} Y_{k, c} / W_{k}$, tabulated during the FGM tables generation.

\section{Configuration and computational setup}

\subsection{ECN spray A}

The investigated spray combustion cases are experiments in ECN Spray A baseline conditions as listed in Table 2. The detailed experimental data are from Sandia [46] 
and CMT [47] for non-reacting and reacting cases respectively. The injection profile was adopted from a "Virtual Injection Rate Generator" [48], as suggested by the ECN.

Table 2. ECN Spray-A operating conditions [16].

\begin{tabular}{ll}
\hline Parameter & Quantity \\
\hline Injector number & $210677 \& 210675$ \\
Institution & Sandia \& CMT \\
Fuel & n-dodecane \\
Nozzle diameter (mm) & 0.09 \\
Discharge coefficient & $0.89 \& 0.91$ \\
Fuel temperature (K) & 363 \\
Injection pressure (MPa) & 150 \\
Injection duration (ms) & 6 \\
Ambient pressure (MPa) & 6 \\
Ambient temperature (K) & 900 \\
Ambient mixture $(\%)^{\mathrm{a}}$ & $\mathrm{N}_{2}-89.71 ; \mathrm{CO}_{2}-6.52 ; \mathrm{H}_{2} \mathrm{O}-3.77$ \\
& $\mathrm{~N}_{2}-84.9 ; \mathrm{O}_{2}-15.1$ \\
\hline
\end{tabular}

${ }^{\mathrm{a}}$ Mole fraction.

\subsection{Computational setup}

In the current study, a new solver based on a standard spray solver from the opensource CFD framework OpenFOAM [49] was developed. New libraries have been created for the FGM storage and retrieval algorithms and are dynamically linked to a customized solver for spray combustion. The gas phase is described in Eulerian framework with the Unsteady Reynolds-averaged NavierStokes (URANS) formulation. Pressure and velocity equations are coupled by the PIMPLE algorithm, which is a combination of the well-known PISO (Pressure Implicit with Splitting of Operator) [50] and SIMPLE (Semi-Implicit Method for Pressure-Linked Equations) [51] algorithms and ensures both stability and accuracy. The liquid phase is presumed to be a discrete phase consisting of individual parcels and treated using Lagrangian particle tracking (LPT). A validation of the flow and combustion models of this new solver with spray combustion flame is reported in [40]. And the FGM solver with memory reduction method predicts similar results with former FGM model while requires much less RAM [43]. This new method enables the usage of detailed mechanisms, such as the one (255 species and 1509 reactions) proposed by Narayanaswamy et al. [20], which is used in the current study. A non-uniform 3D mesh (Fig. 1) refined locally along the spray is created to define the computational domain for a cubical shaped constant volume chamber, with the size of $108 \mathrm{~mm}$ on each side. The time step was set to $5 \times 10^{-7} \mathrm{~s}$, as recommended for RANS simulation by the ECN. The simulation end time was set to $1.5 \mathrm{~ms}$, when the quasi-steady flame is formed. The details of the turbulence and spray sub-models are summarized in Table 3.

Table 3. Related sub-models applied in the simulations.

\begin{tabular}{ll}
\hline Phenomenon & Model \\
\hline Turbulence & $k-\epsilon[52]$ \\
Injection & Blob [53] \\
Breakup & KH-RT [54, 55] \\
Dispersion & Stochastic [56] \\
Particle forces & Spherical Drag [57] \\
Evaporation & Spalding [58] \\
Heat Transfer & Ranz-Marshall [44, 45] \\
\hline
\end{tabular}




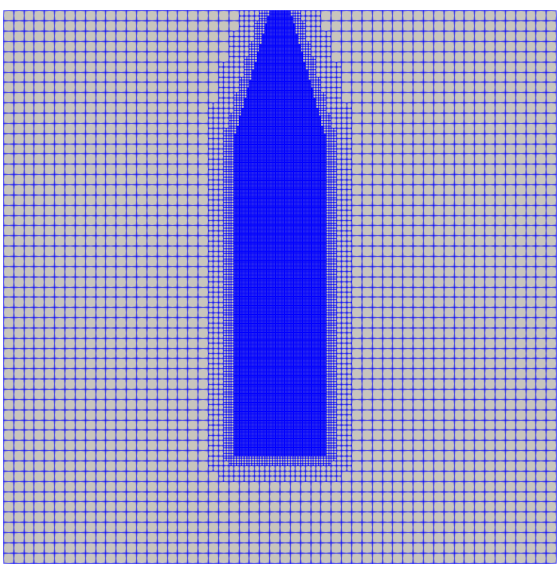

(a)

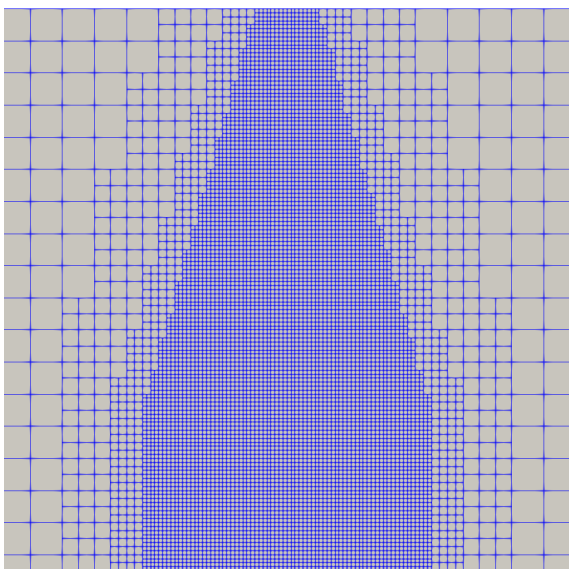

(b)

Figure 1. (a) Computational mesh $(0.25 \mathrm{~mm})$ and (b) local zoom.

\section{Results}

\subsection{Validation for the non-reacting spray case}

Lagrangian particle tracking (LPT) method have been extensively adopted to highpressure spray simulations due to their relative ease of implementation and low computational load. However, many reports pointed out that the accuracy of the EulerianLagrangian spray simulations have a strong dependency on grid size $[59,60,61]$. It's believed that coarse meshes are not able to correctly describe the interaction between the liquid and gas phase causing an underestimation of the spray penetration [59]. To guarantee mesh independence, five mesh resolutions are applied in the current study with minimum cell sizes of $0.125,0.25,0.5,1$ and $2 \mathrm{~mm}$ and respective cell counts of $9081414,1444631,321376,172962$ and 157464 . These five resolutions are obtained through 5 different levels of local refinement along the spray evolution applied to the base grids $(2 \mathrm{~mm})$ in order to properly resolve the flow details. A visualization of the computational mesh $(0.25 \mathrm{~mm})$ is given in Fig. 1, showing the refinement levels and local details. Fig. 2 gives the predicted spray liquid and vapor penetrations with the five grid resolutions. As observed in this figure, longer liquid penetrations are predicted by finer meshes, and the last two refinement levels have similar performance with only little discrepancy at the beginning of spray evolution. Vapor penetration behaves similarly. In consideration of both accuracy and efficiency, the $0.25 \mathrm{~mm}$ mesh was chosen in the current study.

In order to evaluate the present turbulence and spray model setups, the non-reacting Spray A baseline case $\left(0 \% \mathrm{O}_{2}\right)$ is validated against the experimental liquid and vapor penetration as well as fuel mass distribution data. Fig. 3 shows the computed and experimental results of liquid and vapor penetration lengths versus time after the start of injection (ASI). The recommended definitions from ECN are employed to calculate the liquid and vapor penetration lengths. As proposed by the ECN, liquid and vapor penetration are defined as the maximum distance from the nozzle outlet to the farthest axial locations where the liquid volume and vapor mass fraction reach $0.1 \%$. As shown in Fig. 3, liquid and vapor penetration lengths are well predicted compared to experimental trends, with only minor deviations for the vapor penetration at the early stage of injection. Fig. 4 shows the axial and radial distributions of mixture fraction, in 


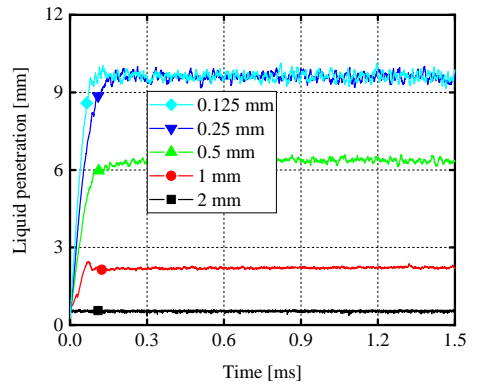

(a) Liquid penetration

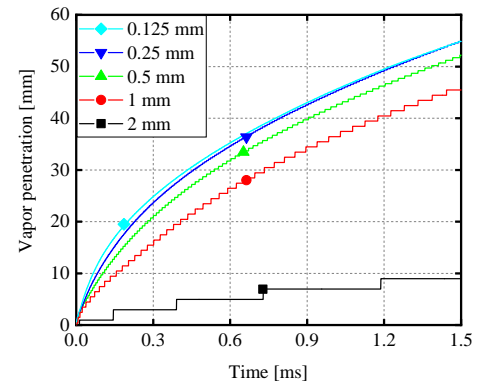

(b) Vapor penetration

Figure 2. Computed spray liquid (a) and vapor (b) penetrations for the five tested grid resolutions.

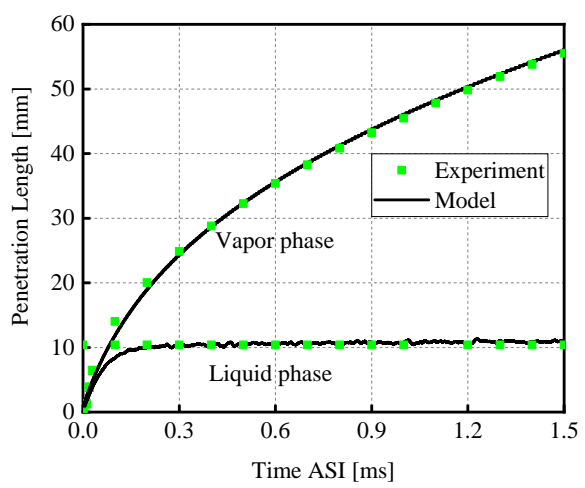

Figure 3. Predictions of the liquid and vapor penetrations under non-reacting condition.

which the radial profiles are sampled at 20 and $40 \mathrm{~mm}$ downstream the injector. In Fig. 4 , the shaded area around the mean profiles corresponds to the standard deviation of the measured mixture fraction. The figure shows the predicted profiles of mean mixture fraction in fairly good agreement with the measured value in the experiment. The difference between the predicted and measured mean mixture fraction is well within the uncertainty range of the experimental results, except for the radial periphery of the spray $40 \mathrm{~mm}$ away from the injector exit, where the mixture fraction is slightly under-predicted.

\subsection{Pre-processing for FGM tables}

The FGM method shares the idea with flamelet approaches [23] that a multidimensional turbulent flame may be considered as an ensemble of stretched onedimensional laminar flames, called flamelets, embedded within the turbulent flow field. It is well-known that there is a diffusion flame surrounding the spray in the conventional diesel engine combustion [1]. Locally the structure of this diffusion flame can be identified as a one-dimensional non-premixed flame. Based on this, 1D counterflow non-premixed laminar flames were solved using FlameMaster [62] according to the unity Lewis number assumption (equal diffusivities for all species and temperature). To build the FGM database, unsteady diffusion flames at a single stoichiometric To 


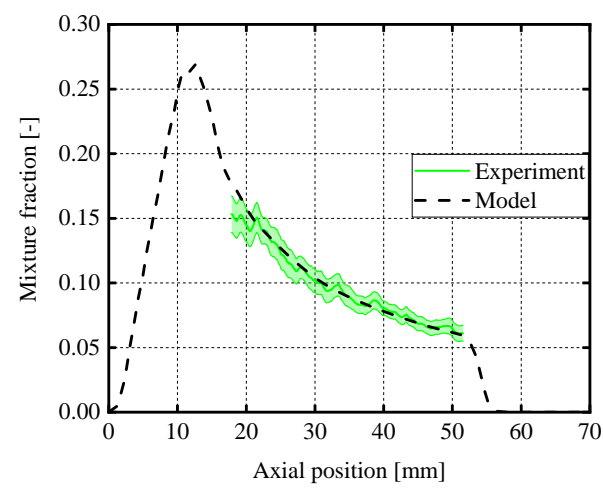

(a) axial

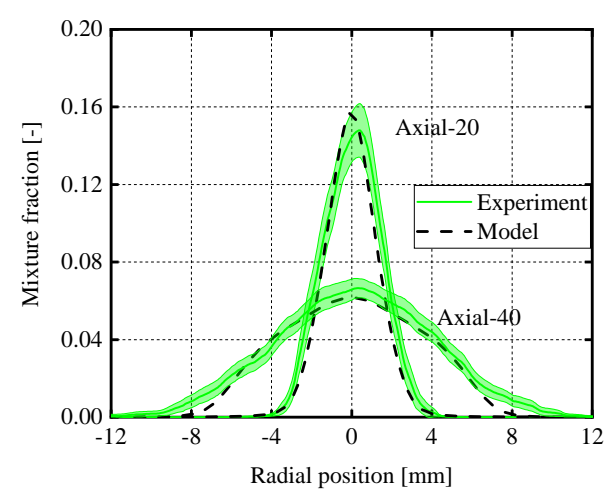

(b) radial

Figure 4. (a)Axial- and (b)radial-profiles of experimental and computed mixture fraction distributions

evaluate the scalar dissipation rate for unsteady ignition process, a series of unsteady flamelets at different scalar dissipation rates are calculated for the spray A baseline case, as shown in Fig. 5a. In Fig. 5b, the ignition delay time (IDT) $\tau_{i g}$ as function of scalar dissipation rate $\chi_{Z, s t}$ is presented. Here, $\tau_{i g}$ is evaluated at stoichiometric condition, and is defined as:

$$
\tau_{i g}=\left\{t \mid T_{s t}(t)=\left(T_{s t}^{0}+T_{s t}^{\infty}\right) / 2\right\}
$$

It is seen that the IDT is quite insensitive to the stoichiometric scalar dissipation rates when $\chi_{Z, s t}<1 \mathrm{~s}^{-1}$. Finally, a single stoichiometric scalar dissipation rate $\left(\chi_{Z, s t}=1 \mathrm{~s}^{-1}\right)$ is chosen for unsteady ignition process to account for the influence of diffusion and transport processes during auto-ignition process, which was validated by Bekdemir [63]. Laminar diffusion flame solutions are dependent on strain rate [64],

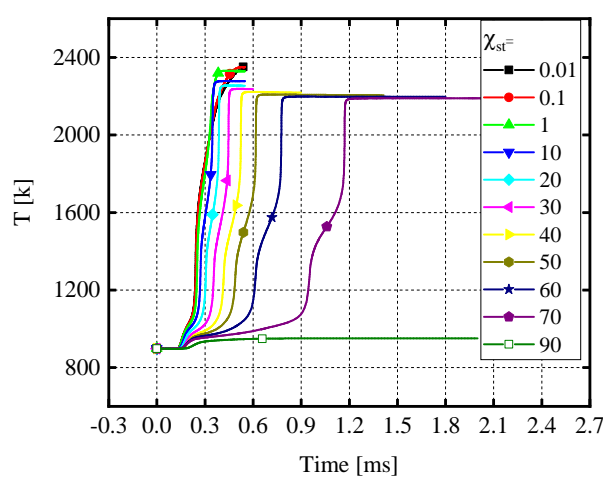

(a)

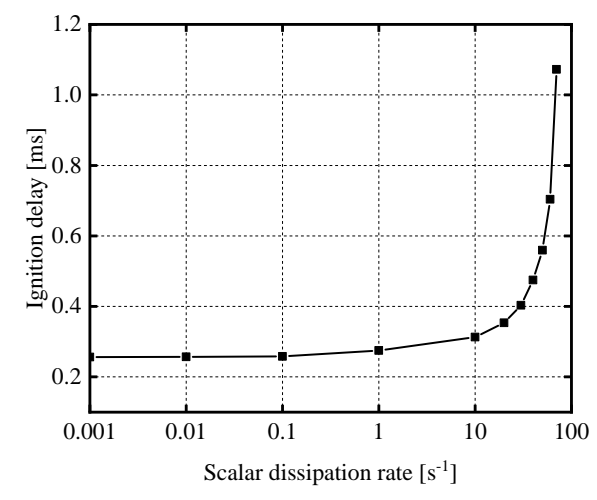

(b)

Figure 5. The effect of scalar dissipation rate on ignition delay in $1 \mathrm{D}$ simulation.

therefore a series of steady flamelets $\left(0.000001 \ldots 1 \mathrm{~s}^{-1}\right)$ is included in the FGM procedure. This approach to generate the FGM tables has also been found to yield good results in several previous researches $[10,11,12,35,63,65]$. The unsteady and steady 
solutions together make up the laminar flamelet database $\phi\left(Z, \chi_{Z, s t}\right)$. Unfortunately, the maximum of progress variable from unsteady flamelet slightly exceeds the steady state value under some mixture fractions. To ensure monotonicity of the progress variable from pure mixing to chemical equilibrium state, the part of unsteady flamelet solution is omitted following Wehrfritz et al. [11, 12]. Another treatment was also tested, that is, deleting the overlapping part of steady flamelet solution, which causes same results like the former method. After the normalization of progress variable according the Eq. 13 and space transformation we get $\phi(Z, C)$. After integration over the presumed PDFs for $Z$ and $C$, we get the turbulent FGM database $\widetilde{\phi}\left(\widetilde{Z}, \widetilde{C}, \widetilde{\zeta_{Z}}, \widetilde{\zeta_{C}}\right)$. The enthalpy loss effect is imposed by decreasing boundary temperature (enthalpy) at the oxidizer side [40]. In the current study, 900, 850, 800, 750 and $700 \mathrm{~K}$ are used for the temperature boundary condition of air side. In Fig 6, progress variable source

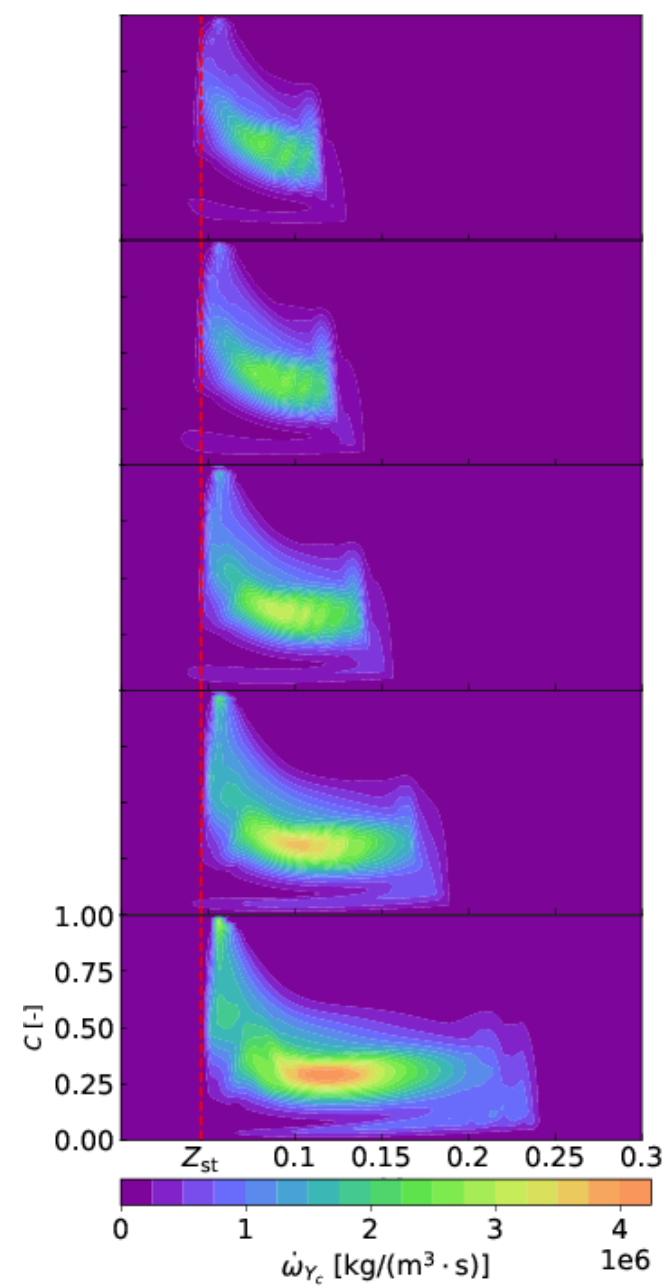

Figure 6. The distribution of progress variable source term in the $Z-C$ space. The five rows data denote results under $700,750,800,850$ and $900 \mathrm{~K}$ respectively.

term on the $Z-C$ space under different air side temperature are presented. It is observed that with air side temperature increasing, the main reaction zone expands to richer region in mixture fraction coordinate and to smaller $C$ in progress variable coordinate. Furthermore, the fastest production rate of reaction progress variable is also inclined to occur in richer mixture fraction regions, and the peak of $\dot{\omega}_{Y_{c}}$ increased, 
under higher air side temperature. There are no direct information of ignition delay time, instead, it depends on the value of progress variable source term. This suggests that reaction will be depressed and occurs in leaner regions under low temperature due to evaporation heat loss, which is neglected in most researches related to spray combustion [10, 11, 12, 35, 63, 65].

After calculation of these five cases under different air side temperature, we get five layers for $\widetilde{\eta_{h}}$, the last dimension of FGM tables $\widetilde{\phi}\left(\widetilde{Z}, \widetilde{C}, \widetilde{\zeta_{Z}}, \widetilde{\zeta_{C}}, \widetilde{\eta_{h}}\right)$. In the CFD solver, transport equations are solved to get these control variables $\widetilde{Z}, \widetilde{Y_{c}}, \widetilde{Z^{\prime \prime 2}}, \widetilde{Y_{c}^{\prime \prime 2}}$, $\widetilde{h}$. Using Eq. 20, 21 and 22, we get $\widetilde{Z}, \widetilde{C}, \widetilde{\zeta_{Z}}, \widetilde{\zeta_{C}}, \widetilde{\eta_{h}} .141$ data points are used for the discretization of mixture fraction, refined around the stoichiometric mixture fraction value. The progress variable has 161 data points and a quadratic spacing is applied (refined towards the mixing limit). 41 uniform levels are considered for $\widetilde{\zeta_{Z}}$ and 21 for $\widetilde{\zeta_{C}} .5$ levels are considered for the last coordinate $\widetilde{\eta_{h}}$.

\subsection{Global view of ignition}

To display the global ignition process, the scatter points colored by the progress variable source term $\overline{\dot{\omega}_{Y_{c}}}$ and colored by the mass fraction of $\mathrm{CH}_{2} \mathrm{O}$ and $\mathrm{OH}$ are presented in the mixture fraction and temperature space at different ignition stages. As shown in Fig. 7, at the early stage of spray evolution $(t=0.2 \mathrm{~ms})$, temperatures distribute almost along the adiabatic mixing line due to evaporation and fuel-air mixing. No significant temperature increase was found. But still progress variable source term are found to arise in the fuel-lean zone, which indicates the occurrence of early reactions. This period prior to auto-ignition is the ignition delay period, and its duration is affected by the local fuel-air mixture and scalar dissipation rate [66]. The second row shows that at $\mathrm{t}=0.3 \mathrm{~ms}$, temperatures near the stoichiometric mixture fraction line $\left(Z_{\text {st }}=0.0463242\right)$ begin to exceed local adiabatic temperature significantly. $\mathrm{CH}_{2} \mathrm{O}$ appears near $Z_{\text {st }}$, and progress variable source term is two orders of magnitude more than that at $\mathrm{t}=0.2 \mathrm{~ms}$. In the third row of Fig. 7b, it is observed that $\mathrm{CH}_{2} \mathrm{O}$ is transported into richer mixture fraction regions, which is the so-called cool flame propagation [4]. And temperatures increase all over the whole reaction zone, reaching its maximum near $Z=0.1$ at 0.45 ms (row 4), which is the so-called second-stage ignition. It is also observed that the $\mathrm{OH}$ concentration is significantly increased near the stoichiometric conditions. The high temperature zone moves towards $Z_{\text {st }}$ line, and even leaner mixture fraction regions. Finally, a quasi-steady high temperature diffusion flame is formed, in which $\mathrm{CH}_{2} \mathrm{O}$ appears mostly in rich regions and $\mathrm{OH}$ concentrates near $Z_{\text {st }}$ line, maintaining the stabilization of the lifted flame. By this stage there are many scatter particles close to the equilibrium line (not shown in the figure), particularly around $Z_{\text {st }}$ line.

\subsection{Effect of TCI on ignition process}

To elucidate the influence of $\beta$-PDF of $Z$ and $C$, two additional cases were conducted, in which $\delta$-PDF is used for $Z$ and $C$ respectively. The ignition delay time (IDT) of these three cases are in Table 4 , where $\beta_{Z}-\beta_{C}$ is the baseline case, meaning $\beta$-PDF is used for $Z$ and $C$ and $\delta_{Z}-\beta_{C}$ means $\delta$-PDF is used for $Z$, and $\beta_{Z}-\delta_{C}$ means $\delta$-PDF is used for $C$. The ignition delay time investigated is defined as the time of maximum gradient in temperature. Applying $\beta$-PDF means that variance should be included 


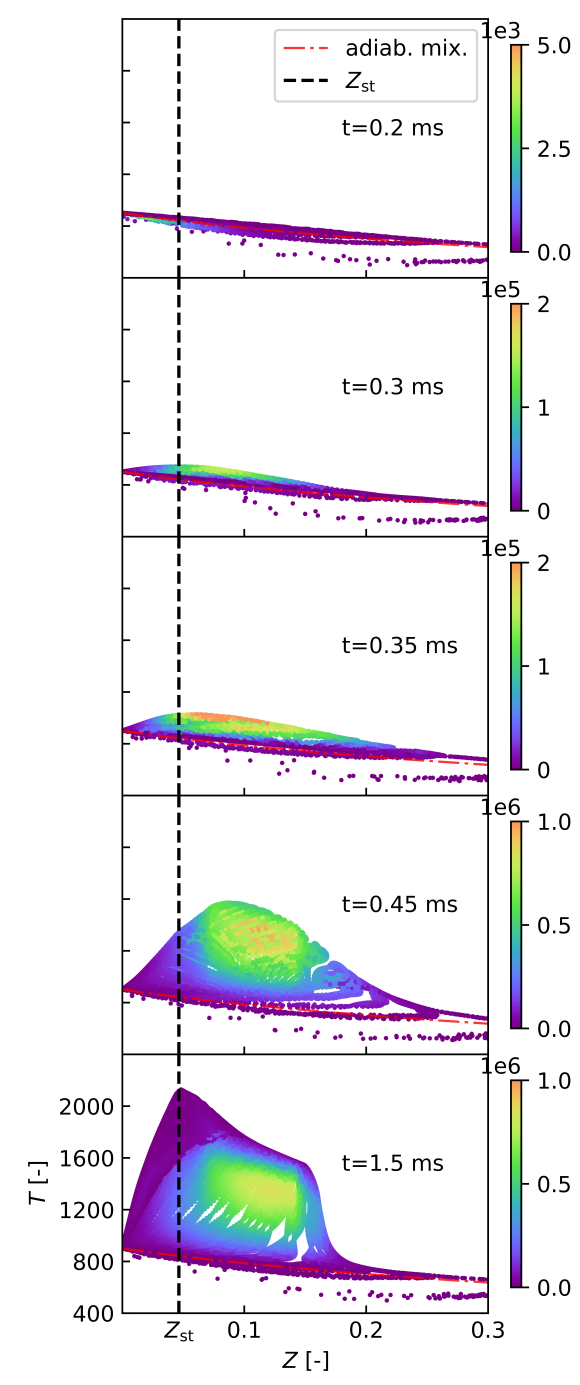

(a)

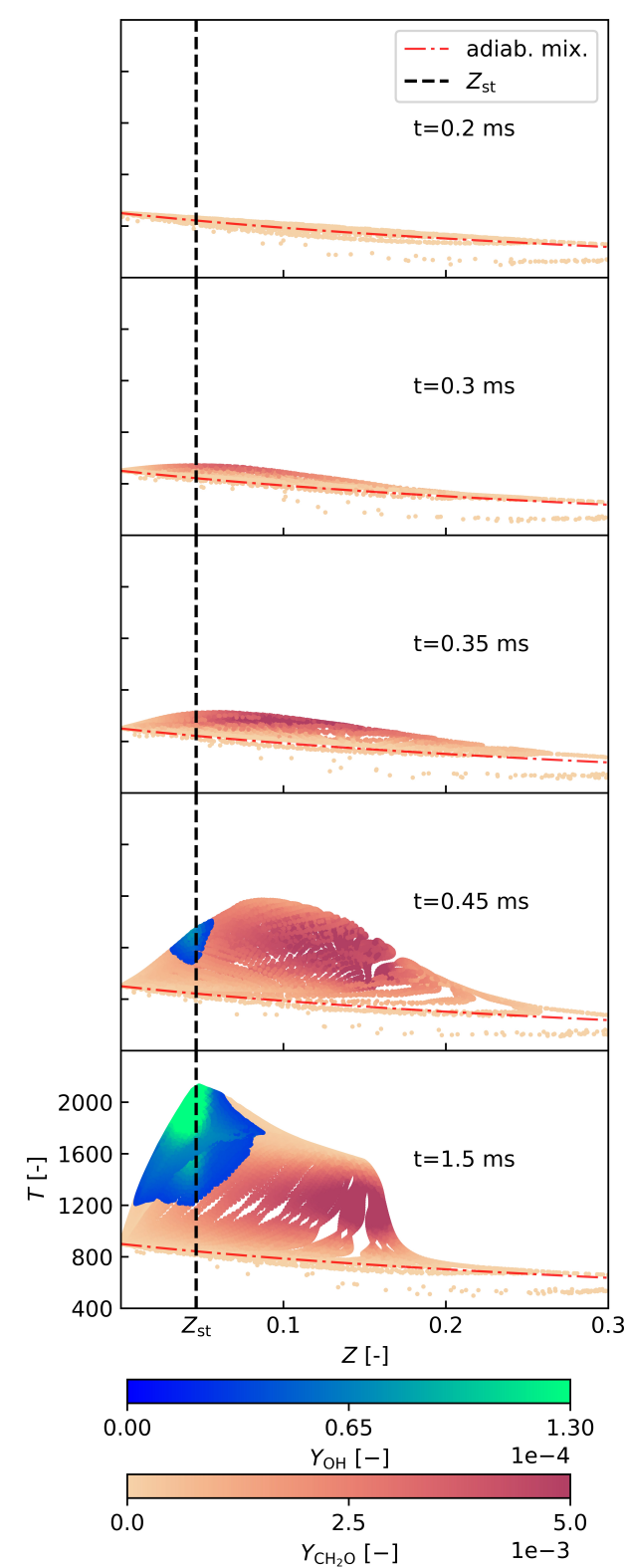

(b)

Figure 7. Scatter point representation in ZT-plane, (a) colored by $\overline{\dot{\omega}_{Y_{c}}}$, (b) colored by $\mathrm{CH}_{2} \mathrm{O}$ and superimposed by $\mathrm{OH}>2 \%$. The red line marks the adiabatic mixing line. The black line marks $Z_{\text {st }}$ line. 
Table 4. Ignition delay times of the three investigated cases and experimental data.

\begin{tabular}{ccccc}
\hline case & $\beta_{Z}-\beta_{C}$ & $\delta_{Z}-\beta_{C}$ & $\beta_{Z}-\delta_{C}$ & experiment \\
\hline IDT & $0.4525 \mathrm{~ms}$ & $0.412 \mathrm{~ms}$ & $0.464 \mathrm{~ms}$ & $0.435 \mathrm{~ms}$ \\
\hline
\end{tabular}

in order to calculate PDF, while variance is not considered in $\delta$-PDF. Table 4 shows that ignition delay changes under the existence of variances of mixture fraction and reaction progress variable. Compared to the baseline case, when $\delta$-PDF is used for $Z$, IDT become shorter; on the contrast, when $\delta$-PDF is used for $C$, IDT will be slightly longer. That is to say, ignition is retarded by the consideration of $Z$ variance, while it is promoted by $C$ variance.

In 3D solver, the most important variable interpolated from FGM tables is the progress variable source term $\overline{\dot{\omega}_{Y_{c}}}$, which determines the reaction progress and ignition delay time.

$$
\overline{\dot{\omega}_{Y_{c}}}=\frac{\dot{\omega}_{C O_{2}}}{M_{C O_{2}}}+\frac{\dot{\omega}_{C O}}{M_{C O}}+\frac{\dot{\omega}_{H O_{2}}}{M_{H O_{2}}}
$$

Hence, in order to explain the influence of these variances, the temporal evolution of $\overline{\dot{\omega}_{Y_{c}}}$ changes over $\widetilde{Z}$ in the cases of $\beta_{Z-} \beta_{C}, \delta_{Z-} \beta_{C}$ and $\beta_{Z}-\delta_{C}$ is presented in Fig. 8. It is observed that when $\delta$-PDF is applied for $Z$ (case $\delta_{Z}-\beta_{C}$ ), $\overline{\dot{\omega}}_{Y_{c}}$ is much larger than that in the baseline case at any moment and its distribution along the mixture fraction coordinate gets narrower. And $\overline{\dot{\omega}_{Y_{c}}}$ in the $\beta_{Z} \delta_{C}$ case is smaller than that in the baseline case at early times $(0.4 \mathrm{~ms})$ while much larger at steady states $(1.5 \mathrm{~ms})$. Despite the small difference in global ignition characteristics (ignition delay time) between the case $\beta_{Z}-\beta_{C}$ and $\beta_{Z}-\delta_{C}$, the discrepancies are more pronounced in the temporal evolution of reaction progress variable source term. The distinction is more clear in the figure of progress variable source term maximum over time (Fig. 9a). It is obvious that, before auto-ignition, progress variable source term in the case $\delta_{Z}-\beta_{C}$ is higher, and that in the case $\beta_{Z}-\delta_{C}$ is lower, compared with the baseline case. So it is not strange that the case $\delta_{Z}-\beta_{C}$ ignites earlier and the case $\beta_{Z^{-}} \delta_{C}$ ignites later by comparison with baseline case. The mixture fraction in the cell where progress variable source term peaks are extracted, and its temporal evolution is presented in Fig. 9b. As shown in Fig 9b, at the beginning of reaction progress, $\overline{\dot{\omega}_{Y_{c}}}$ peaks at fuel-lean regions, and then transfers to richer zones, finally reaches the steady maximum. This is inline with the global ignition process described in the section 4.3. According to the $1 \mathrm{D}$ flamelets results (distribution of progress variable source term in $Z-C$ space) shown in Fig. $6, \overline{\dot{\omega}_{Y_{c}}}$ peaks at leaner regions under lower air side temperature. So it's not strange that early emergence of progress variable source term occurs at lean regions since evaporation heat loss is considered in the current study. From Fig. 9b, it is also observed that the mixture fraction where progress variable source term peaks increases with time and becomes stable at approximately $\widetilde{Z}=0.11$ when the flame gets steady in the three cases. In addition, the figure shows the mixture fraction of case $\delta_{Z}-\beta_{C}$ is 0.02 higher than that of baseline case at any time during the initial transient phase, which could be caused by the shorter ignition delay. From Fig 6 , it is clear that the progress variable source term is monotonically increasing at $\widetilde{Z}<0.11$. Since $\widetilde{Z}$ in the case $\delta_{Z}-\beta_{C}$ is higher, its progress variable source term will also higher. So this is the reason why neglecting variance of mixture fraction will shorten the ignition delay time.

As for variance of progress variable, it is shown in Fig 9a that neglect of variance of 


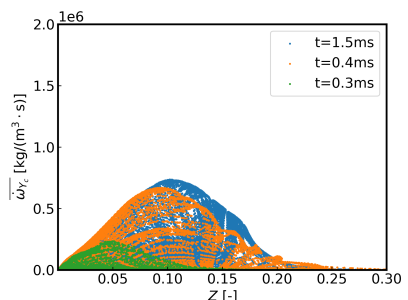

(a) $\beta_{Z}-\beta_{C}$

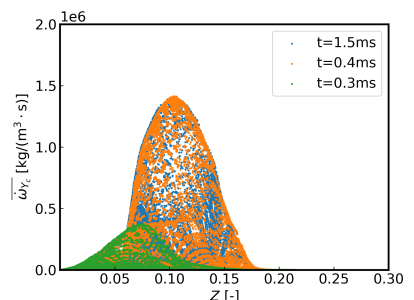

(b) $\delta_{Z}-\beta_{C}$

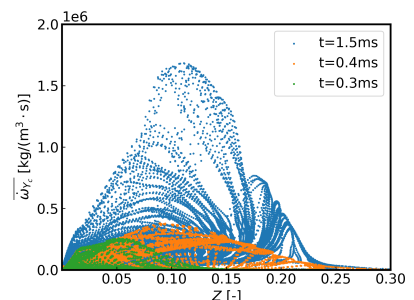

(c) $\beta_{Z}-\delta_{C}$

Figure 8. The evolution of progress variable source term as a function of $Z$ for the three cases.

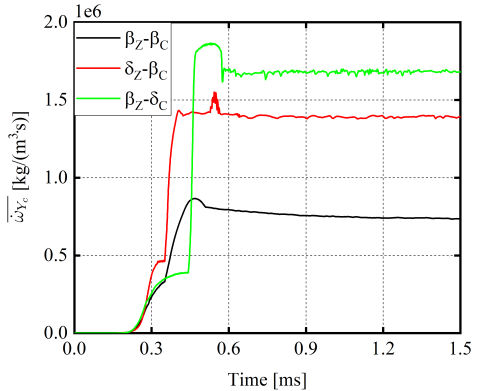

(a)

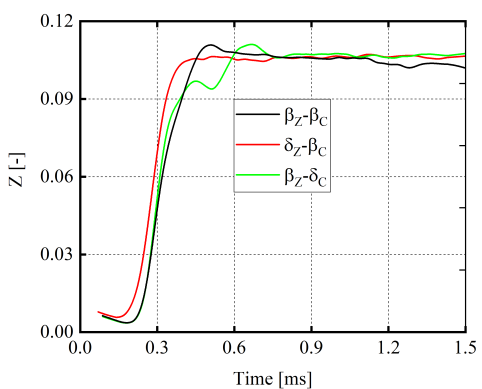

(b)

Figure 9. The time history of reaction progress variable source term maximum (a) and the corresponding $\widetilde{Z}$ for the three cases (b). (neglect of early times when $\overline{\dot{\omega}_{Y_{c}}}$ are smaller than 1)

progress variable will cause that temporal evolution of progress variable source term shows three stages, that is, slightly higher and then lower and finally at quasi-steady state much higher than that of baseline case. At early times (around $0.3 \mathrm{~ms}$ ), in the cell where progress variable source term peaks, the mixture fraction and progress variable are the same for the $\beta_{Z}-\delta_{C}$ and $\beta_{Z}-\beta_{C}$ cases. So it is the existence of variance of progress variable that causes the different $\overline{\dot{\omega}_{Y_{c}}}$. We extract $\widetilde{Z}, \widetilde{C}, \widetilde{\zeta_{Z}}, \widetilde{\zeta_{C}}$ and $\widetilde{\eta_{h}}$ in the cell where progress variable source term reaches its maximum at $0.3 \mathrm{~ms}, 0.36 \mathrm{~ms}$ and $1.5 \mathrm{~ms}$, corresponding the three stages above. Since the variance of progress variable is investigated here, $\widetilde{\zeta_{Z}}$ and $\widetilde{\eta_{h}}$ are assumed to be zero. Fig. 10 gives progress variable source term as a function of progress variable under the three stages, the black line is the turbulent value of $\overline{\dot{\omega}_{Y_{c}}}$ using $\delta$-PDF for progress variable and the red line is that of using $\beta$-PDF under the $\widetilde{\zeta_{C}}$ in the cell where progress variable source term peaks. It is seen from Fig. 10 that $\bar{\omega}_{Y_{c}}$ has a bimodal distribution along the $C$ coordinate. When $\widetilde{C}$ is near the peak value, $\overline{\dot{\omega}_{Y_{c}}}$ of $\delta$-PDF is bigger than that of $\beta$-PDF, as shown in Fig $10 \mathrm{a}$ and $10 \mathrm{c}$ when $\mathrm{t}=0.3 \mathrm{~ms}$ and $\mathrm{t}=1.5 \mathrm{~ms}$. And when $\widetilde{C}$ is in the valley of the distribution of $\overline{\dot{\omega}_{Y_{c}}}$, the turbulent value of $\beta$-PDF is bigger than that of $\delta$-PDF (see Fig. 10b).

The reason of these different results considering variance or not is analyzed below. Taking distribution of progress variable source term when $\widetilde{C}=0.1$ (see Fig. 11) as an example. Especially, the black line $\left(\widetilde{\zeta_{Z}}=0\right)$ denotes the case of $\delta$-PDF, and also denotes the laminar distribution on $Z$. Let the mixture fraction where $\dot{\omega}_{Y_{c}}$ peaks be $Z_{\max }$ from the laminar solution. Then the $\delta$-PDF turbulent value peaks at $Z_{\max }$ too. 


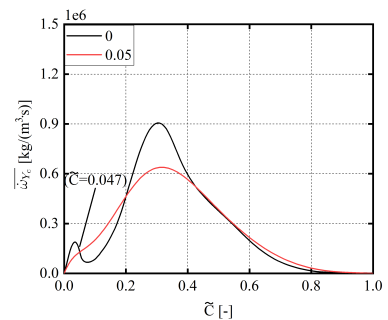

(a) $\mathrm{t}=0.3 \mathrm{~ms}, \widetilde{Z}=0.05$

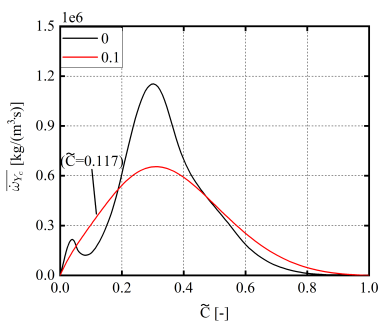

(b) $\mathrm{t}=0.36 \mathrm{~ms}, \widetilde{Z}=0.077$

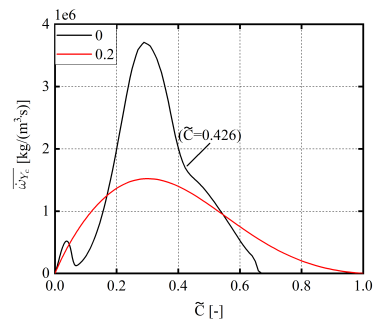

(c) $\mathrm{t}=1.5 \mathrm{~ms}, \widetilde{Z}=0.1$

Figure 10. $\overline{\dot{\omega}_{Y_{c}}}$ profiles from FGM lookup tables under three key moments. The black line marks $\delta_{C}$. The red line marks $\beta_{C}$.

While the $\beta$-PDF turbulent value will be depressed near the peak since the presence of variance. However, in the regions of $\widetilde{Z}>0.2$, the $\delta$-PDF turbulent value is very small. Unlike $\delta$-PDF, in the $\beta$-PDF integration process, the turbulent value will be supplemented from where progress variable source term is relatively larger. This explains the distribution of $\overline{\dot{\omega}_{Y_{c}}}$ along mixture fraction under different shapes of PDF distribution.

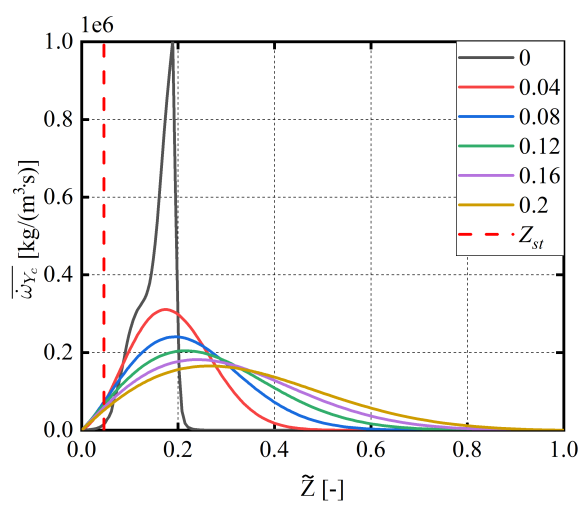

Figure 11. The distribution of progress variable source term over mixture fraction under different $\widetilde{\zeta_{Z}}$.

To analysis the effect of variance to heat release in the ignition process, heat release rate (HRR) is included into the FGM table. In FlameMaster, HRR is calculated as: $\sum_{i} \dot{\omega}_{i} \cdot h_{i}$, where $\omega_{i}$ is the reaction rate of specie $i$, and $h_{i}$ is the absolute enthalpy of specie $i$. In CFD solver, heat release rate is integrated over the whole domain in each time step. As shown in Fig. 12, the HRR in the case of baseline and $\beta_{Z}-\delta_{C}$ is similar with the experimental data. The case $\delta_{Z}-\beta_{C}$ severely underestimates the $\mathrm{HRR}$, although there is little discrepancy in the ignition delay. That is to say, $\beta$-PDF is quite necessary for the integration of mixture fraction to get a reasonable heat release rate. On the other hand, the case $\beta_{Z}-\delta_{C}$ predicts highest HRR in the ignition process which is closest to the experiment. But the baseline case predicts best result in the subsequent process. 


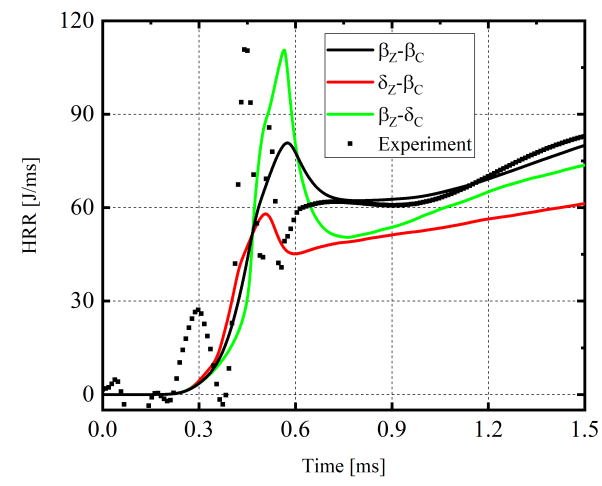

Figure 12. Time evolution of the heat release rate in the combustion chamber.

\subsection{Effect of TCI on flame structure}

Furthermore, it is found that the variance of mixture fraction and progress variable will also change the flame structure in the steady state condition. Fig. 13 shows the computed $\mathrm{OH}$ mass fraction distributions at $1.5 \mathrm{~ms}$ ASI from the results obtained by the cases $\beta_{Z^{-}} \beta_{C}, \delta_{Z^{-}} \beta_{C}$ and $\beta_{Z^{-}} \delta_{C}$. At first, we talk about the effect of variance of mixture fraction. As shown in Fig. 13, there is a significant discrepancy in $\mathrm{OH}$ mass fraction distribution between $\beta_{Z}-\beta_{C}$ and $\delta_{Z}-\beta_{C}$ cases. Peak values of $\mathrm{OH}$ mass fraction are much lower in the case of $\beta$-PDF for $Z$, compared to those from $\delta$-PDF. On the other hand, $\beta$-PDF, which takes variance of mixture fraction into account, predicts more distributed $\mathrm{OH}$ mass fraction in space. While the flame for the $\delta$-PDF is relatively thinner. The same findings were reported in other ECN spray(n-heptane or n-dodecane) combustion modelings using mRIF, FGM, TFM and TPDF models $[10,17,8,7,18]$, attributing to turbulent fluctuations in these researches. As for the influence of variance of progress variable, the distribution of $\mathrm{OH}$ mass fraction in Fig. 13 shows that the flame structures and the $\mathrm{OH}$ mass fraction maximums are similar between the case $\beta_{Z^{-}} \beta_{C}$ and the case $\beta_{Z^{-}} \delta_{C}$. That is to say, variance of progress variable has little influence over flame structure. Nevertheless, it makes flame liftoff length shorter, obviously seen from Fig. 13c. The similar impact of variance of progress variable was found in a lifted methane/air auto-ignition flame simulation using an unsteady flamelet/progress variable model [67].

In what follows, above phenomena are also found to be caused by the characteristic of $\beta$-PDF. As seen from the $\mathrm{OH}$ mass fraction contour in Fig. 13, $\mathrm{OH}$ is distributed mainly around the $Z_{\text {st }}$ contour line. To investigate the distribution of integrated value of $\mathrm{OH}$ mass fraction in the FGM tables at steady state, the turbulent values of $\mathrm{OH}$ mass fraction from FGM tables under different $\widetilde{\zeta_{Z}}$ when $\widetilde{C}=1$ is presented in Fig. 14a. As we know, the PDF becomes Dirac $\delta$ shape when $\widetilde{\zeta_{Z}}$ is zero, so the black solid line denotes the turbulent $\widetilde{Y_{\mathrm{OH}}}$ integrated using $\delta$-PDF. When $\delta$-PDF is used for $Z$, it is shown that $\widetilde{Y_{\mathrm{OH}}}$ near $Z_{\mathrm{st}}$ is much higher than that of $\beta$-PDF, but the span of $\widetilde{Y_{\mathrm{OH}}}$ in mixture fraction direction is very narrow. While for $\beta$-PDF, the maximum of $\widetilde{Y_{\mathrm{OH}}}$ is largely suppressed due to the introduction of variance of mixture fraction and the distribution along $\widetilde{Z}$ coordinate is more dispersing, also more suppression and broader range for higher $\widetilde{\zeta_{Z}}$. So if $\beta$-PDF is used to generate the FGM tables, in CFD solver, the maximum will be much less than that of $\delta$-PDF, while the range in mixture 
fraction is broader.

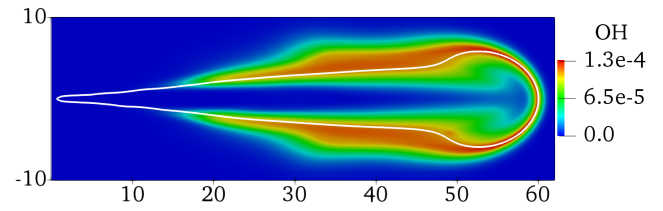

(a) $\beta_{Z}-\beta_{C}$

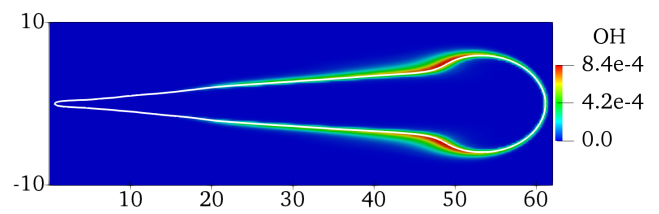

(b) $\delta_{Z}-\beta_{C}$

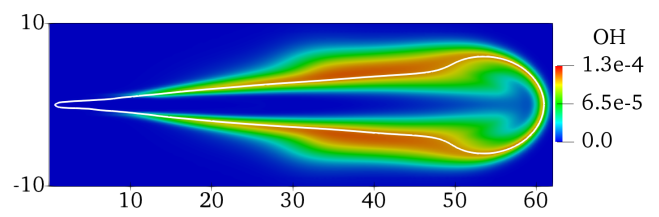

(c) $\beta_{Z}-\delta_{C}$

Figure 13. The $\mathrm{OH}$ mass fraction contours under the three cases at $\mathrm{t}=1.5 \mathrm{~ms}$.

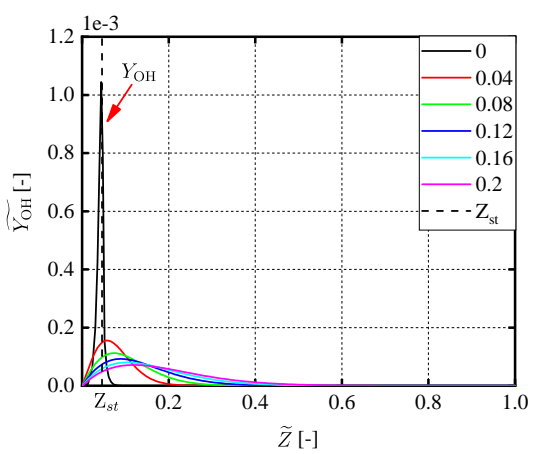

(a)

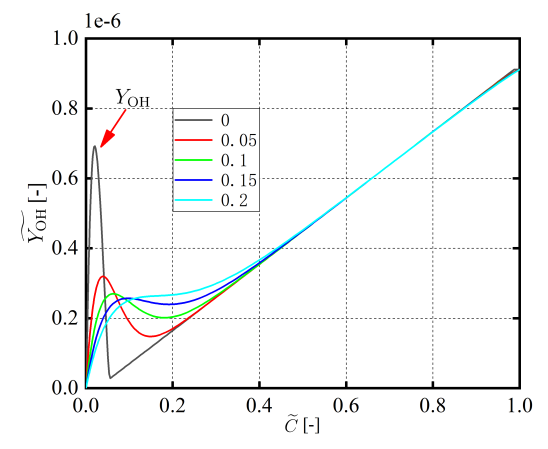

(b)

Figure 14. The mass fraction of $\mathrm{OH}$ in FGM tables under different $\widetilde{\zeta_{Z}}$ when $\widetilde{C}=1$ (a) and the mass fraction of $\mathrm{OH}$ in FGM tables under different $\widetilde{\zeta_{C}}$ when $\widetilde{Z}=0.01$ (b).

To find out the reason of variance of progress variable shortens the lift-off length, the turbulent value of $\mathrm{OH}$ mass fraction over progress variable under $\widetilde{Z}=0.01$ is plotted in Fig. 14b. Since at lift-off position, OH is distributed near the spray core radial periphery where mixture fraction is quite small. As can be seen in Fig. 14b, the black line indicates the $\mathrm{OH}$ value under $\delta$-PDF, which is higher than the $\beta$-PDF values under small progress variables. This phenomenon is also attributed to the effect of $\beta$-PDF as discussed above. 


\section{Conclusions}

RANS modelling of ECN spray A baseline condition have been carried out using a recently developed FGM combustion model, which allows application of detailed chemical mechanisms in spray combustion simulations. The non-reacting case was initially investigated to validate the mesh resolution and the spray sub-models. A mesh resolution with minimum cell size of $0.25 \mathrm{~mm}$ is found to obtain a good agreement with experiments respect to liquid and vapor penetrations and mixture fraction distribution. During preparation of FGM tables with enthalpy deficit, we found that source term of reaction progress variable decreases with decline of temperature due to evaporation heat loss. In addition, the maximum region of progress variable source term moves to leaner condition when temperature of air side decreases. The global ignition process shows that early reaction happens on the lean side, and the first-stage ignition initiates close to the stoichiometric conditions and propagates to a richer mixture and promotes the ignition there, which featured cool flame propagation. Then second stage ignition occurs on the rich side. Finally, a steady diffusion flame formed, and the steady flame structure was investigated further.

The variance of mixture fraction has already been highlighted by many researchers. In the current study, we found that neglect of variance of mixture fraction will shorten ignition delay time and cause a very thin flame structure. Furthermore, peak value of $\mathrm{OH}$ mass fraction is largely depressed with including of variance of mixture fraction. We found that it is $\beta$-PDF that cause the broad distribution of $\mathrm{OH}$ mass fraction. The distribution of progress variable source term along the mixture fraction coordinate is unimodal under different progress variable, and so is that of $\mathrm{OH}$ mass fraction. After applying $\beta$-PDF for mixture fraction, the maximum will be distributed into other regions and the whole distribution becomes broader. Furthermore, it is found that variance of progress variable, which is often neglected in spray combustion modelling due to the mixing controlled feature, has also an impact on auto-ignition and the flame structure. We found that neglect of variance of progress variable will retard auto-ignition and shorten flame lift-off length.

\section{Acknowledgement(s)}

The work was carried out at National Supercomputer Center in Tianjin, and the calculations were performed on TianHe-1(A). Our deepest gratitude goes to the editor and the anonymous reviewers for their careful work and thoughtful suggestions that have helped improve the manuscript substantially.

\section{Disclosure statement}

No potential conflict of interest was reported by the authors.

\section{Funding}

This work was supported by Major Research Plan of the National Natural Science Foundation of China (No. 91541205); National Natural Science Foundation of China [grant numbers 51876140]; the project of National Key R\&D Program of China 
(2017YFE0102800); This project has also received funding from the European Union's Horizon 2020 research and innovation programme under the Marie Skłodowska-Curie grant agreement No. 713673. Ambrus Both has received financial support through the "la Caixa" INPhINIT Fellowship Grant for Doctoral studies at Spanish Research Centres of Excellence, "la Caixa" Banking Foundation, Barcelona, Spain.

\section{References}

\section{References}

[1] J.E. Dec, A Conceptual Model of DI Diesel Combustion Based on Laser-Sheet Imaging, in SAE Technical Paper. 970873, 1997.

[2] P. Kundu, M.M. Ameen, and S. Som, Importance of turbulence-chemistry interactions at low temperature engine conditions, Combust. Flame 183 (2017), pp. 283-298.

[3] T. Poinsot and D. Veynante, Theoretical and numerical combustion, 2nd ed., Edwards, Philadelphia, 2005.

[4] R.N. Dahms, G.A. Paczko, S.A. Skeen, and L.M. Pickett, Understanding the ignition mechanism of high-pressure spray flames, Proc. Combust. Inst. (2016).

[5] Y. Pei, E.R. Hawkes, M. Bolla, S. Kook, G.M. Goldin, Y. Yang, S.B. Pope, and S. Som, An analysis of the structure of an n-dodecane spray flame using TPDF modelling, Combust. Flame 168 (2016), pp. 420-435.

[6] M. Bolla, M.A. Chishty, E.R. Hawkes, Q.N. Chan, and S. Kook, Influence of turbulent fluctuations on radiation heat transfer, NO and soot formation under ECN Spray A conditions, Proc. Combust. Inst. (2016).

[7] P. Kundu, Y. Pei, M. Wang, R. Mandhapati, and S. Som, Evaluation of turbulence-chemistry interaction under diesel engine conditions with multiflamelet RIF model, Atomization Sprays 24 (2014), pp. 779-800.

[8] G. D'Errico, T. Lucchini, F. Contino, M. Jangi, and X.S. Bai, Comparison of well-mixed and multiple representative interactive flamelet approaches for diesel spray combustion modelling, Combust. Theory Model. 18 (2014), pp. 65-88.

[9] C. Bajaj, M. Ameen, and J. Abraham, Evaluation of an Unsteady Flamelet Progress Variable Model for Autoignition and Flame Lift-Off in Diesel Jets, Combust. Sci. Technol. 185 (2013), pp. 454-472.

[10] U. Egüz, S. Ayyapureddi, C. Bekdemir, B. Somers, and P. de Goey, Manifold resolution study of the FGM method for an igniting diesel spray, Fuel 113 (2013), pp. 228-238.

[11] A. Wehrfritz, O. Kaario, V. Vuorinen, and B. Somers, Large Eddy Simulation of $n$-dodecane spray flames using Flamelet Generated Manifolds, Combust. Flame 167 (2016), pp. 113-131.

[12] H. Kahila, A. Wehrfritz, O. Kaario, M. Ghaderi Masouleh, N. Maes, B. Somers, and V. Vuorinen, Large-eddy simulation on the influence of injection pressure in reacting Spray A, Combust. Flame 191 (2018), pp. 142-159.

[13] M.M. Ameen, P. Kundu, and S. Som, Novel Tabulated Combustion Model Approach for Lifted Spray Flames with Large Eddy Simulations, SAE Int. J. Engines 9 (2016), pp. 2056-2065.

[14] P. Kundu, M.M. Ameen, C. Xu, U. Unnikrishnan, T. Lu, and S. Som, Implementation of Detailed Chemistry Mechanisms in Engine Simulations, in ASME 2017 Internal Combustion Engine Division Fall Technical Conference. American 
Society of Mechanical Engineers, 2017, pp. V002T06A016-V002T06A016.

[15] C.K. Westbrook, W.J. Pitz, O. Herbinet, H.J. Curran, and E.J. Silke, A comprehensive detailed chemical kinetic reaction mechanism for combustion of n-alkane hydrocarbons from n-octane to n-hexadecane, Combust. Flame 156 (2009), pp. 181-199.

[16] Engine Combustion Network, https://ecn.sandia.gov.

[17] Y. Pei, E.R. Hawkes, and S. Kook, Transported probability density function modelling of the vapour phase of an n-heptane jet at diesel engine conditions, Proc. Combust. Inst. 34 (2013), pp. 3039-3047.

[18] P. Kundu, M. Ameen, U. Unnikrishnan, and S. Som, Implementation of a Tabulated Flamelet Model for Compression Ignition Engine Applications, in SAE Technical Paper. 2017-01-0564, 2017.

[19] E. Ranzi, A. Frassoldati, A. Stagni, M. Pelucchi, A. Cuoci, and T. Faravelli, Reduced Kinetic Schemes of Complex Reaction Systems: Fossil and Biomass-Derived Transportation Fuels, Int. J. Chem. Kinet. 46 (2014), pp. 512-542.

[20] K. Narayanaswamy, P. Pepiot, and H. Pitsch, A chemical mechanism for low to high temperature oxidation of n-dodecane as a component of transportation fuel surrogates, Combust. Flame 161 (2014), pp. 866-884.

[21] A.C. Hindmarsh, ODEPACK, a systematized collection of ODE solvers, Scientific Computing (1983), pp. 55-64.

[22] Z. Luo, S. Som, S.M. Sarathy, M. Plomer, W.J. Pitz, D.E. Longman, and T. $\mathrm{Lu}$, Development and validation of an $n$-dodecane skeletal mechanism for spray combustion applications, Combust. Theory Modell. 18 (2014), pp. 187-203.

[23] N. Peters, Laminar diffusion flamelet models in non-premixed turbulent combustion, Prog. Energy Combust. Sci. 10 (1984), pp. 319-339.

[24] N. Peters, Turbulent combustion, Cambridge monographs on mechanics, Cambridge university press, Cambridge, 2000.

[25] H. Barths, C. Hasse, G. Bikas, and N. Peters, Simulation of combustion in direct injection diesel engines using a eulerian particle flamelet model, Proc. Combust. Inst. 28 (2000), pp. 1161-1168.

[26] J.B. Michel and O. Colin, A tabulated diffusion flame model applied to diesel engine simulations, Int. J. Engine Res. 15 (2013), pp. 346-369.

[27] J.A. van Oijen and L.D.E. GOEY, Modelling of Premixed Laminar Flames using Flamelet-Generated Manifolds, Combust. Sci. Technol. 161 (2000), pp. 113-137.

[28] M. Ihme, C.M. Cha, and H. Pitsch, Prediction of local extinction and re-ignition effects in non-premixed turbulent combustion using a flamelet/progress variable approach, Proc. Combust. Inst. 30 (2005), pp. 793-800.

[29] L. Vervisch, R. Hauguel, P. Domingo, and M. Rullaud, Three facets of turbulent combustion modelling: DNS of premixed $V$-flame, LES of lifted nonpremixed flame and RANS of jet-flame, J. Turbul. 5 (2004), pp. 1-8.

[30] C. Bekdemir, L.M. Somers, and L.P. de Goey, First application of the flamelet generated manifold (FGM) approach to the simulation of an igniting diesel spray, in 19th International Multidimensional Engine Modeling User's Group Meeting at the SAE Congress, Detroit, Michigan. 2009.

[31] G. Sorrentino, M. de Joannon, P. Sabia, R. Ragucci, and A. Cavaliere, Numerical investigation of the ignition and annihilation of $c h 4 / n 2 / 02$ mixtures under mild operative conditions with detailed chemistry, Combustion Theory and Modelling 21 (2016), pp. 120-136.

[32] S.B. Pope, Turbulent flows, Cambridge university press, Cambridge, 2000.

[33] R.W. Bilger, S.H. Stårner, and R.J. Kee, On reduced mechanisms for methane-air 
combustion in nonpremixed flames, Combust. Flame 80 (1990), pp. 135-149.

[34] M. Ihme, L. Shunn, and J. Zhang, Regularization of reaction progress variable for application to flamelet-based combustion models, J. Comput. Phys. 231 (2012), pp. $7715-7721$.

[35] C. Bekdemir, L. Somers, L. de Goey, J. Tillou, and C. Angelberger, Predicting diesel combustion characteristics with Large-Eddy Simulations including tabulated chemical kinetics, Proc. Combust. Inst. 34 (2013), pp. 3067-3074.

[36] C. Bekdemir, B. Somers, and P. de Goey, DNS with detailed and tabulated chemistry of engine relevant igniting systems, Combust. Flame 161 (2014), pp. 210-221.

[37] M. Blocquet, C. Schoemaecker, D. Amedro, O. Herbinet, F. Battin-Leclerc, and C. Fittschen, Quantification of $\mathrm{OH}$ and HO2 radicals during the low-temperature oxidation of hydrocarbons by Fluorescence Assay by Gas Expansion technique, Proc. Natl. Acad. Sci. 110 (2013), pp. 20014-20017.

[38] H. Wang, K. Luo, and J. Fan, Direct numerical simulation and CMC (conditional moment closure) sub-model validation of spray combustion, Energy 46 (2012), pp. 606-617.

[39] S. Mukhopadhyay and J. Abraham, Evaluation of an unsteady flamelet progress variable model for autoignition and flame development in compositionally stratified mixtures, Physics of Fluids 24 (2012), p. 075115.

[40] L. Ma and D. Roekaerts, Modeling of spray jet flame under MILD condition with non-adiabatic FGM and a new conditional droplet injection model, Combust. Flame 165 (2016), pp. 402-423.

[41] G. Kuenne, A. Ketelheun, and J. Janicka, LES modeling of premixed combustion using a thickened flame approach coupled with FGM tabulated chemistry, Combust. Flame 158 (2011), pp. 1750-1767.

[42] E. Knudsen, Shashank, and H. Pitsch, Modeling partially premixed combustion behavior in multiphase LES, Combust. Flame 162 (2015), pp. 159-180.

[43] A Both, RANS-FGM simulation of n-heptane spray flame in OpenFOAM, Master's thesis, Delft University of Technology, 2017.

[44] W.E. Ranz, W.R. Marshall, et al., Evaporation from drops, Part I, Chem. Eng. Prog 48 (1952), pp. 141-146.

[45] W.E. Ranz, W.R. Marshall, et al., Evaporation from drops, Part II, Chem. Eng. Prog 48 (1952), pp. 173-180.

[46] L.M. Pickett, J. Manin, C.L. Genzale, D.L. Siebers, M.P.B. Musculus, and C.A. Idicheria, Relationship Between Diesel Fuel Spray Vapor Penetration/Dispersion and Local Fuel Mixture Fraction, SAE Int. J. Engines 4 (2011), pp. 764-799.

[47] J. Benajes, R. Payri, M. Bardi, and P. Martí-Aldaraví, Experimental characterization of diesel ignition and lift-off length using a single-hole ECN injector, Appl. Therm. Eng. 58 (2013), pp. 554-563.

[48] Universitat Politecnica de Valencia CMT Virtual Injection Rate Generator, Available online at URL http://www.cmt.upv.es/ECN03.aspx\#model.

[49] H.G. Weller, G. Tabor, H. Jasak, and C. Fureby, A tensorial approach to computational continuum mechanics using object-oriented techniques, Comput. Phys. 12 (1998), p. 620.

[50] R. Issa, Solution of the implicitly discretised fluid flow equations by operatorsplitting, J. Comput. Phys. 62 (1986), pp. 40-65.

[51] S. Patankar, Numerical heat transfer and fluid flow, CRC press, 1980.

[52] W.P. Jones and B. Launder, The prediction of laminarization with a two-equation model of turbulence, Int. J. Heat Mass Transfer 15 (1972), pp. 301-314.

[53] R.D. Reitz, Modeling atomization processes in high-pressure vaporizing sprays, 
Atomisation Spray Technol. 3 (1987), pp. 309-337.

[54] R.D. Reitz and R. Diwakar, Structure of High-Pressure Fuel Sprays, in SAE Technical Paper. 870598, 1987.

[55] R.D. Reitz and J.C. Beale, Modeling spray atomization with the KelvinHelmholtz/Rayleigh-Taylor hybrid model, Atomization Sprays 9 (1999), pp. 623650.

[56] P.J. O'Rourke, Statistical properties and numerical implementation of a model for droplet dispersion in a turbulent gas, J. Comput. Phys. 83 (1989), pp. 345-360.

[57] A.A. Amsden, P.J. O'rourke, and T.D. Butler, KIVA-II: A computer program for chemically reactive flows with sprays, Tech. Rep. LA-11560-MS, Los Alamos National Lab., NM (USA).

[58] D.B. Spalding, The combustion of liquid fuels, in Symp. (Int.) Combust., Vol. 4. 1953, pp. 847-864.

[59] T. Lucchini, G. D'Errico, and D. Ettorre, Numerical investigation of the spraymesh-turbulence interactions for high-pressure, evaporating sprays at engine conditions, Int. J. Heat Fluid Flow 32 (2011), pp. 285-297.

[60] P.K. Senecal, E. Pomraning, K.J. Richards, and S. Som, An investigation of grid convergence for spray simulations using an LES turbulence model, in SAE Technical Paper. 2013-01-1083, 2013.

[61] A. Wehrfritz, V. Vuorinen, O. Kaario, and M. Larmi, Large eddy simulation of high-velocity fuel sprays: studying mesh resolution and breakup model effects for spray A, Atomization Sprays 23 (2013), pp. 419-442.

[62] H. Pitsch, FlameMaster: A C++ computer program for $0 D$ combustion and $1 D$ laminar flame calculations (1998). Available at https://www.itv.rwthaachen.de/index.php?id=13.

[63] C. Bekdemir, L. Somers, and L. de Goey, Modeling diesel engine combustion using pressure dependent Flamelet Generated Manifolds, Proc. Combust. Inst. 33 (2011), pp. 2887-2894.

[64] E. Mastorakos, Ignition of turbulent non-premixed flames, Prog. Energy Combust. Sci. 35 (2009), pp. 57-97.

[65] U. Egüz, N. Leermakers, B. Somers, and P. de Goey, Modeling of PCCI combustion with FGM tabulated chemistry, Fuel 118 (2014), pp. 91-99.

[66] S. Mukhopadhyay and J. Abraham, Influence of compositional stratification on autoignition in n-heptane/air mixtures, Combust. Flame 158 (2011), pp. 10641075.

[67] M. Ihme and Y.C. See, Prediction of autoignition in a lifted methane/air flame using an unsteady flamelet/progress variable model, Combust. Flame 157 (2010), pp. 1850-1862. 